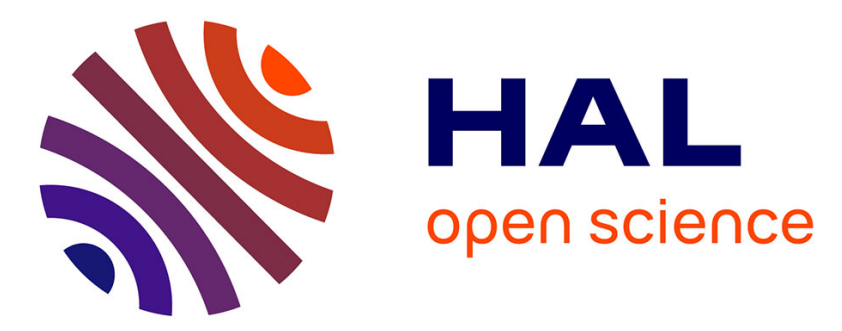

\title{
Resolution of the implicit Euler scheme for the Navier-Stokes equation through a least-squares method
}

Arnaud Munch, Jérôme Lemoine

\section{To cite this version:}

Arnaud Munch, Jérôme Lemoine. Resolution of the implicit Euler scheme for the Navier-Stokes equation through a least-squares method. 2020. hal-01996429v3

\section{HAL Id: hal-01996429 \\ https://hal.uca.fr/hal-01996429v3}

Preprint submitted on 10 Jul 2020 (v3), last revised 29 Dec 2020 (v4)

HAL is a multi-disciplinary open access archive for the deposit and dissemination of scientific research documents, whether they are published or not. The documents may come from teaching and research institutions in France or abroad, or from public or private research centers.
L'archive ouverte pluridisciplinaire HAL, est destinée au dépôt et à la diffusion de documents scientifiques de niveau recherche, publiés ou non, émanant des établissements d'enseignement et de recherche français ou étrangers, des laboratoires publics ou privés. 


\title{
RESOLUTION OF THE IMPLICIT EULER SCHEME FOR THE NAVIER-STOKES EQUATION THROUGH A LEAST-SQUARES METHOD
}

\author{
JÉRÔME LEMOINE AND ARNAUD MÜNCH
}

\begin{abstract}
This work analyzes a least-squares method in order to solve implicit time schemes associated to the 2D and 3D Navier-Stokes system, introduced in 1979 by Bristeau, Glowinksi, Periaux, Perrier and Pironneau. Implicit time schemes reduce the numerical resolution of the Navier-Stokes system to multiple resolutions of steady Navier-Stokes equations. We first construct a minimizing sequence (by a gradient type method) for the least-squares functional which converges strongly and quadratically toward a solution of a steady Navier-Stokes equation from any initial guess. The method turns out to be related to the globally convergent damped Newton approach applied to the Navier-Stokes operator, in contrast to standard Newton method used to solve the weak formulation of the Navier-Stokes system. Then, we apply iteratively the analysis on the fully implicit Euler scheme and show the convergence of the method uniformly with respect to the time discretization. Numerical experiments for 2D examples support our analysis.
\end{abstract}

Key Words. Steady Navier-Stokes equation, Implicit time scheme, Least-squares approach, Damped Newton method.

AMS subject classifications. 35Q30, 93E24.

\section{Introduction - Motivation}

Let $\Omega \subset \mathbb{R}^{d}, d=2$ or $d=3$ be a bounded connected open set whose boundary $\partial \Omega$ is Lipschitz and $T>0$. We denote by $\mathcal{V}=\left\{v \in \mathcal{D}(\Omega)^{d}, \nabla \cdot v=0\right\}, \boldsymbol{H}$ the closure of $\mathcal{V}$ in $L^{2}(\Omega)^{d}$ and $\boldsymbol{V}$ the closure of $\mathcal{V}$ in $H^{1}(\Omega)^{d}$, endowed with the norm $\|v\|_{\boldsymbol{V}}=\|\nabla v\|_{2}$.

The Navier-Stokes system describes a viscous incompressible fluid flow in the bounded domain $\Omega$ during the time interval $(0, T)$ submitted to the external force $F$. It reads as follows :

$$
\left\{\begin{array}{l}
u_{t}-\nu \Delta u+(u \cdot \nabla) u+\nabla p=F, \quad \nabla \cdot u=0 \text { in } \Omega \times(0, T), \\
u=0 \text { on } \partial \Omega \times(0, T), \\
u(\cdot, 0)=u_{0} \text { in } \Omega,
\end{array}\right.
$$

where $u$ is the velocity of the fluid, $p$ its pressure and $\nu$ is the viscosity constant assumed smaller than one. We refer to [19. This work is concerned with the numerical approximation of 1.1 through the time marching fully implicit Euler scheme

$$
\left\{\begin{array}{l}
y^{0}=u_{0} \quad \text { in } \Omega, \\
\frac{y^{n+1}-y^{n}}{\delta t}-\nu \Delta y^{n+1}+\left(y^{n+1} \cdot \nabla\right) y^{n+1}+\nabla \pi^{n+1}=\frac{1}{\delta t} \int_{t_{n}}^{t_{n+1}} F(\cdot, s) d s, \quad n \geq 0, \\
\nabla \cdot y^{n+1}=0 \quad \text { in } \Omega, \quad n \geq 0, \\
y^{n+1}=0 \quad \text { on } \partial \Omega, \quad n \geq 0,
\end{array}\right.
$$

Date: July 8, 2020.

Laboratoire de Mathématiques, Université Clermont Auvergne, UMR CNRS 6620, Campus des Cézeaux, 63177 Aubière, France. e-mail: jerome.lemoine@uca.fr.

Laboratoire de Mathématiques, Université Clermont Auvergne, UMR CNRS 6620, Campus des Cézeaux, 63177 Aubière, France. e-mail: arnaud.munch@uca.fr. 
where $\left\{t_{n}\right\}_{n=0 \ldots N}$, for a given $N \in \mathbb{N}$, is a uniform discretization of the time interval $(0, T)$. $\delta t=T / N$ is the time discretization step. This also-called backward Euler scheme is studied for instance in [19, chapter 3, section 4]. It is proved there that the piecewise linear interpolation (in time) of $\left\{y^{n}\right\}_{n \in[0, N]}$ weakly converges in $L^{2}(0, T, \boldsymbol{V})$ toward a solution $u$ of 1.1 as $\delta t$ goes to zero. It achieves a first order convergence with respect to $\delta t$. We also refer to [20] for a stability analysis of the scheme in long time. We refer to [17] and the references therein for Crank-Nicolson schemes achieving second order convergence.

The determination of $y^{n+1}$ from $y^{n}$ requires the resolution of a nonlinear partial differential equation. Precisely $y^{n+1}$ together with the pressure $\pi^{n+1}$ solve the following problem: find $y \in \boldsymbol{V}$ and $\pi \in L_{0}^{2}(\Omega)$, solution of

$$
\left\{\begin{array}{l}
\alpha y-\nu \Delta y+(y \cdot \nabla) y+\nabla \pi=f+\alpha g, \quad \nabla \cdot y=0 \quad \text { in } \Omega, \\
y=0 \quad \text { on } \partial \Omega,
\end{array}\right.
$$

with

$$
\alpha:=\frac{1}{\delta t}>0, \quad f:=\frac{1}{\delta t} \int_{t_{n}}^{t_{n+1}} F(\cdot, s) d s, \quad g=y^{n} .
$$

Recall that for any $f \in H^{-1}(\Omega)^{d}$ and $g \in L^{2}(\Omega)^{d}$, there exists one solution $(y, \pi) \in \boldsymbol{V} \times L_{0}^{2}(\Omega)$ of 1.3. unique if $\|g\|_{L^{2}(\Omega)^{d}}^{2}+\alpha^{-1} \nu^{-1}\|f\|_{H^{-1}(\Omega)^{d}}^{2}$ is small enough (see Proposition 2.3 for a precise statement). $L_{0}^{2}(\Omega)$ stands for the space of functions in $L^{2}(\Omega)^{d}$ with zero means.

A weak solution $y \in \boldsymbol{V}$ of 1.3 solves the formulation $F(y, w)=0$ for all $w \in \boldsymbol{V}$ where $F$ is defined by

$$
\begin{aligned}
F(y, z):=\int_{\Omega} \alpha y \cdot z+ & \nu \nabla y \cdot \nabla z+(y \cdot \nabla) y \cdot z \\
& \quad<f, z>_{H^{-1}(\Omega)^{d} \times H_{0}^{1}(\Omega)^{d}}-\alpha \int_{\Omega} g \cdot z=0, \quad \forall z \in \boldsymbol{V} .
\end{aligned}
$$

If $D_{y} F$ is invertible, one may approximate a weak solution through the iterative Newton method: a sequence $\left\{y_{k}\right\}_{k} \in \boldsymbol{V}$ is constructed as follows

$$
\left\{\begin{array}{l}
y_{0} \in \boldsymbol{V}, \\
D_{y} F\left(y_{k}, w\right) \cdot\left(y_{k+1}-y_{k}\right)=-F\left(y_{k}, w\right), \quad \forall w \in \boldsymbol{V}, k \geq 0 .
\end{array}\right.
$$

If the initial guess $y_{0}$ is close enough to a weak solution of $(1.3)$, i.e. a solution satisfying $F(y, w)=0$ for all $w$, then the sequence $\left\{y_{k}\right\}_{k}$ converges. We refer to [15, Section 10.3] and [4, Chapter 6]) and for some numerical aspects to [10].

Alternatively, we may also employ least-squares methods which consists in minimizing a quadratic functional, which measure how an element $y$ is close to the solution. For instance, we may introduce the extremal problem : $\inf _{y \in \boldsymbol{V}} E(y)$ with $E: \boldsymbol{V} \rightarrow \mathbb{R}^{+}$defined by

$$
E(y):=\frac{1}{2} \int_{\Omega} \alpha|v|^{2}+\nu|\nabla v|^{2}
$$

where the corrector $v$, together with the pressure, is the unique solution in $\boldsymbol{V} \times L_{0}^{2}(\Omega)$ of the linear boundary value problem:

$$
\left\{\begin{array}{l}
\alpha v-\nu \Delta v+\nabla \pi+(\alpha y-\nu \Delta y+(y \cdot \nabla) y-f-\alpha g)=0, \quad \nabla \cdot v=0 \quad \text { in } \quad \Omega \\
v=0 \quad \text { on } \partial \Omega
\end{array}\right.
$$

Remark that $E(y)$ vanishes if and only if $y \in \boldsymbol{V}$ is a weak solution of 1.3 , equivalently a zero of $F(y, w)=0$ for all $w \in \boldsymbol{V}$. As a matter of fact, the infimum is reached. Least-squares methods to solve nonlinear boundary value problems have been the subject of intensive developments in the last decades, as they present several advantages, notably on computational and stability viewpoints. We refer to the books [1, 17. The minimization of the functional $E$ over $\boldsymbol{V}$ leads 
to a so-called weak least squares method. Precisely, the equality $\sqrt{2 E(y)}=\sup _{w \in \boldsymbol{V}, w \neq 0} \frac{F(y, w)}{\|w\|_{V}}$ shows that $E$ is equivalent to the $\boldsymbol{V}^{\prime}$ norm of the Navier-Stokes equation (we refer to Remark 2.9. . The terminology " $H^{-1}$-least-squares method" is employed in [2] where the minimization of $E$ has been introduced and numerically implemented to approximate the solutions of 1.1 through the scheme (1.2). We also mention [4, Chapter 4, Section 6] which studied later the use of a least-squares strategy to solve a steady Navier-Stokes equation without incompressibility constraint.

The first objective of the present work is to analyze rigorously the method introduced in [2] and show that one may construct minimizing sequences in $\boldsymbol{V}$ for $E$ that converge strongly toward a solution of 1.3 . The second objective is to justify the use of that weak least-squares method to solve iteratively a weak formulation of the scheme 1.2 , leading to an approximation of the solution of (1.1). This requires to show some convergence properties of a minimizing sequence for $E$, uniformly with respect to the parameter $n$ related to the time discretization. As we shall see, this requires smallness assumptions on the data $u_{0}$ and $F$.

The rest of the paper is organized as follows. Section 2 is devoted to the analyze the leastsquares method (1.7)-1.8 associated to weak solutions of $[1.3)$. We show that $E$ is differentiable over $\boldsymbol{V}$ and that any critical point for $E$ in the ball $B$ defined in Definition 2.1 is also a zero of $E$. This is done by introducing a descent direction $Y_{1}$ for $E$ at any point $y \in \boldsymbol{V}$ for which $E^{\prime}(y) \cdot Y_{1}$ is proportional to $E(y)$. Then, assuming that there exists at least one solution of 1.3 in the ball $B$, we show that any minimizing sequence $\left\{y_{k}\right\}_{(k \in \mathbb{N})}$ for $E$ uniformly in $B$ strongly converges to a solution of 2.5. Such limit belongs to $B$ and is actually the unique solution. Eventually, we construct a minimizing sequence (defined in (2.22) based on the element $Y_{1}$ and initialized with $g$ assume in $\boldsymbol{V}$. We show that, if $\alpha$ is large enough, then this particular sequence is uniformly in $B$ and converges (quadratically after a finite number of iterates related to the values of $\nu$ and $\alpha$ ) strongly to the solution of (1.3) (see Theorem 2.15). This specific sequence coincides with the one obtained from the damped Newton method, a globally convergent generalization of 1.6 .

Then, in Section (3), as an application, we consider the least-squares approach to solve iteratively the backward Euler scheme (see (3.1)), weak formulation of 1.2 . For each $n>0$, in order to approximate $y^{n+1}$, we define a minimizing sequence $\left\{y_{k}^{n+1}\right\}_{k \geq 0}$ based on $Y_{1}^{n+1}$ and initialized with $y^{n}$. Adapting the global convergence result of Section 2, we then show, assuming $\left\|u_{0}\right\|_{L^{2}(\Omega)^{d}}+\|F\|_{L^{2}\left(0, T ; H^{-1}(\Omega)^{d}\right)}$ small enough, the strong convergence of the minimizing sequences, uniformly with respect to the time discretization parameter $n$ (see Theorem 3.7). The analysis is performed for $d=2$ for both weak and regular solutions and for $d=3$ for regular solutions. Our analysis justifies the use of Newton type methods to solve implicit time schemes for 1.1, as mentioned in [15, Section 10.3]. To the best of our knowledge, such analysis of convergence is original.

In Section 4, we discuss numerical experiments based on finite element approximations in space for two 2D geometries: the celebrated example of the channel with a backward facing step and the semi-circular driven cavity introduced in [5]. We notably exhibit for small values of the viscosity constant the robustness of the damped Newton method (compared to the Newton one).

\section{Analysis of a Least-Squares method For a Steady NAVIER-Stokes Equation}

We analyse in this section a least-squares method to solve the steady Navier-Stokes equation (1.3) assuming $\alpha>0$ : we extend [1] where the particular case $\alpha=0$ is addressed.

2.1. Technical preliminary results. We endow the space $\boldsymbol{V}$ with the norm $\|y\|_{\boldsymbol{V}}:=\|\nabla y\|_{2}$, for all $y \in \boldsymbol{V} .\|\cdot\|_{2}$ stands for the $L^{2}$ norm $\|\cdot\|_{L^{2}(\Omega) d}$. We shall also use the following notations

$$
\|y\|_{\boldsymbol{V}}^{2}:=\alpha\|y\|_{2}^{2}+\nu\|\nabla y\|_{2}^{2}, \quad \forall y \in \boldsymbol{V}
$$

and $\left\langle y, z>_{\boldsymbol{V}}:=\alpha \int_{\Omega} y \cdot z+\nu \int_{\Omega} \nabla y \cdot \nabla z\right.$ so that $<y, z>_{\boldsymbol{V}} \leq\|y\|_{\boldsymbol{V}}\|z\|_{\boldsymbol{V}}$ for any $y, z \in \boldsymbol{V}$. 
In the sequel, we repeatedly use the following classical estimates (see [19]).

Lemma 2.1. Let any $u, v \in \boldsymbol{V}$. If $d=2$, then

$$
-\int_{\Omega} u \cdot \nabla u \cdot v=\int_{\Omega} u \cdot \nabla v \cdot u \leq \sqrt{2}\|u\|_{2}\|\nabla v\|_{2}\|\nabla u\|_{2} .
$$

If $d=3$, then there exists a constant $c=c(\Omega)$ such that

$$
\int_{\Omega} u \cdot \nabla v \cdot u \leq c\|u\|_{2}^{1 / 2}\|\nabla v\|_{2}\|\nabla u\|_{2}^{3 / 2} .
$$

Definition 2.1. For any $y \in \boldsymbol{V}$, we define

$$
\tau_{d}(y):=\left\{\begin{array}{l}
\frac{\|y\|_{\boldsymbol{V}}}{\sqrt{2 \alpha \nu}}, \quad \text { if } \quad d=2, \\
\frac{M\|y\|_{\boldsymbol{V}}}{\left(\alpha \nu^{3}\right)^{1 / 4}}, \quad \text { if } \quad d=3
\end{array}\right.
$$

with $M:=\frac{3^{3 / 4}}{4} c$ and $c$ from 2.2 .

We shall also repeatedly use the following Young type inequalities.

Lemma 2.2. For any $u, v \in \boldsymbol{V}$, the following inequalities hold true :

$$
\sqrt{2}\|u\|_{2}\|\nabla v\|_{2}\|\nabla u\|_{2} \leq \tau_{2}(v)\|u\|_{\boldsymbol{V}}^{2}
$$

if $d=2$ and

$$
c\|u\|_{2}^{1 / 2}\|\nabla v\|_{2}\|\nabla u\|_{2}^{3 / 2} \leq \tau_{3}(v)\|u\|_{\boldsymbol{V}}^{2}
$$

if $d=3$.

Let $f \in H^{-1}(\Omega)^{d}, g \in L^{2}(\Omega)^{d}$ and $\alpha \in \mathbb{R}_{+}^{\star}$. The weak formulation of 1.3 reads as follows: find $y \in \boldsymbol{V}$ solution of

(2.5) $\alpha \int_{\Omega} y \cdot w+\nu \int_{\Omega} \nabla y \cdot \nabla w+\int_{\Omega} y \cdot \nabla y \cdot w=<f, w>_{H^{-1}(\Omega)^{d} \times H_{0}^{1}(\Omega)^{d}}+\alpha \int_{\Omega} g \cdot w, \quad \forall w \in \boldsymbol{V}$.

The following result holds true (we refer to [13]).

Proposition 2.3. Assume $\Omega \subset \mathbb{R}^{d}$ is bounded and Lipschitz. There exists at least one solution $y$ of 2.5) satisfying

$$
\|y\|_{V}^{2} \leq c_{0} \nu\|f\|_{H^{-1}(\Omega)^{d}}^{2}+\alpha\|g\|_{2}^{2}
$$

where $c_{0}>0$, only connected to the Poincaré constant, depends on $\Omega$. If moreover $\Omega$ is $C^{2}$ and $f \in L^{2}(\Omega)^{d}$, then any solution $y \in \boldsymbol{V}$ of (2.5) belongs to $H^{2}(\Omega)^{d}$.

Lemma 2.4. Assume that a solution $y \in \boldsymbol{V}$ of (2.5) satisfies $\tau_{d}(y)<1$. Then, such solution is the unique solution of 2.5).

Proof. Let $y_{1} \in \boldsymbol{V}$ and $y_{2} \in \boldsymbol{V}$ be two solutions of 2.5). Set $Y=y_{1}-y_{2}$. Then,

$$
\alpha \int_{\Omega} Y \cdot w+\nu \int_{\Omega} \nabla Y \cdot \nabla w+\int_{\Omega} y_{2} \cdot \nabla Y \cdot w+\int_{\Omega} Y \cdot \nabla y_{1} \cdot w=0 \quad \forall w \in V .
$$

We now take $w=Y$ and use that $\int_{\Omega} y_{2} \cdot \nabla Y \cdot Y=0$. If $d=2$, we use 2.1) and 2.3 to get

$$
\|Y\|_{\boldsymbol{V}}^{2}=-\int_{\Omega} Y \cdot \nabla y_{1} \cdot Y \leq \tau_{2}\left(y_{1}\right)\|Y\|_{\boldsymbol{V}}^{2}
$$

leading to $\left(1-\tau_{2}\left(y_{1}\right)\right)\|Y\|_{\boldsymbol{V}}^{2} \leq 0$. Consequently, if $\tau_{2}\left(y_{1}\right)<1$ then $Y=0$ and the solution of 2.5 is unique. In particular, in view of 2.6 , this holds if the data satisfy $\nu\|g\|_{2}^{2}+\frac{c_{0}}{\alpha}\|f\|_{H^{-1}(\Omega)^{d}}^{2}<2 \nu^{3}$. 
If $d=3$, we use 2.2 and $(2.4)$ to obtain

$$
\|Y\|_{\boldsymbol{V}}^{2}=-\int_{\Omega} Y \cdot \nabla y_{1} \cdot Y \leq c\|Y\|_{2}^{\frac{1}{2}}\|\nabla Y\|_{2}^{\frac{3}{2}}\left\|\nabla y_{1}\right\|_{2} \leq \tau_{3}\left(y_{1}\right)\|Y\|_{\boldsymbol{V}}^{2}
$$

leading to $\left(1-\tau_{3}\left(y_{1}\right)\right)\|Y\|_{2}^{2} \leq 0$ and to the uniqueness of the solution if $\tau_{3}\left(y_{1}\right)<1$. In particular, in view of 2.6 , this holds if the data satisfy $\nu\|g\|_{2}^{2}+\frac{c_{0}}{\alpha}\|f\|_{H^{-1}(\Omega)^{d}}^{2}<M^{-2} \nu^{7 / 2} \alpha^{-1 / 2}$.

We now introduce our least-squares functional $E: \boldsymbol{V} \rightarrow \mathbb{R}^{+}$as follows

$$
E(y):=\frac{1}{2} \int_{\Omega}\left(\alpha|v|^{2}+\nu|\nabla v|^{2}\right)=\frac{1}{2}\|v\|_{\boldsymbol{V}}^{2}
$$

where the corrector $v \in \boldsymbol{V}$ is the unique solution of the linear formulation

$$
\begin{aligned}
\alpha \int_{\Omega} v \cdot w+\nu \int_{\Omega} \nabla v \cdot \nabla w=-\alpha & \int_{\Omega} y \cdot w-\nu \int_{\Omega} \nabla y \cdot \nabla w-\int_{\Omega} y \cdot \nabla y \cdot w \\
& +<f, w>_{H^{-1}(\Omega)^{d} \times H_{0}^{1}(\Omega)^{d}}+\alpha \int_{\Omega} g \cdot w, \quad \forall w \in \boldsymbol{V} .
\end{aligned}
$$

In particular, for $d=2$, the corrector $v$ satisfies the estimate:

$$
\|v\|_{\boldsymbol{V}} \leq\|y\|_{\boldsymbol{V}}\left(1+\frac{\|y\|_{\boldsymbol{V}}}{2 \sqrt{\alpha} \nu}\right)+\sqrt{\frac{c_{0}\|f\|_{H^{-1}}^{2}}{\nu}+\alpha\|g\|_{2}^{2}} .
$$

Conversely, we also have

$$
\|y\|_{\boldsymbol{V}} \leq\|v\|_{\boldsymbol{V}}+\sqrt{\frac{c_{0}\|f\|_{H^{-1}}^{2}}{\nu}+\alpha\|g\|_{2}^{2}}
$$

The infimum of $E$ is equal to zero and is reached by a solution of (2.5). In this sense, the functional $E$ is a so-called error functional which measures, through the corrector variable $v$, the deviation of the pair $y$ from being a solution of the underlying equation (2.5).

A practical way of taking a functional to its minimum is through some (clever) use of descent directions, i.e. the use of its derivative. In doing so, the presence of local minima is always something that may dramatically spoil the whole scheme. The unique structural property that discards this possibility is the strict convexity of the functional. However, for non-linear equations like (2.5), one cannot expect this property to hold for the functional $E$ in (2.7). Nevertheless, we insist in that for a descent strategy applied to the extremal problem $\min _{y \in \boldsymbol{V}} E(y)$ numerical procedures cannot converge except to a global minimizer leading $E$ down to zero.

Indeed, we would like to show that the only critical points for $E$ correspond to solutions of 2.5. In such a case, the search for an element $y$ solution of 2.5 is reduced to the minimization of $E$.

For any $y \in \boldsymbol{V}$, we now look for an element $Y_{1} \in \boldsymbol{V}$ solution of the following formulation

$$
\alpha \int_{\Omega} Y_{1} \cdot w+\nu \int_{\Omega} \nabla Y_{1} \cdot \nabla w+\int_{\Omega}\left(y \cdot \nabla Y_{1}+Y_{1} \cdot \nabla y\right) \cdot w=-\alpha \int_{\Omega} v \cdot w-\nu \int_{\Omega} \nabla v \cdot \nabla w, \forall w \in \boldsymbol{V}
$$

where $v \in \boldsymbol{V}$ is the corrector (associated to $y$ ) solution of 2.8. $Y_{1}$ enjoys the following property.

Proposition 2.5. For all $y \in \boldsymbol{V}$ satisfying $\tau_{d}(y)<1$, there exists a unique solution $Y_{1}$ of (2.11) associated to $y$. Moreover, this solution satisfies

$$
\left(1-\tau_{d}(y)\right)\left\|Y_{1}\right\|_{\boldsymbol{V}} \leq \sqrt{2 E(y)} .
$$

Proof. The proof uses the arguments of Lemma 2.4. We define the bilinear and continuous form $a: \boldsymbol{V} \times \boldsymbol{V} \rightarrow \mathbb{R}$ by

$$
a(Y, w)=\alpha \int_{\Omega} Y \cdot w+\nu \int_{\Omega} \nabla Y \cdot \nabla w+\int_{\Omega}(y \cdot \nabla Y+Y \cdot \nabla y) \cdot w
$$


so that $a(Y, Y)=\|Y\|_{\boldsymbol{V}}^{2}+\int_{\Omega} Y \cdot \nabla y \cdot Y$. If $d=2$, using (2.3), we obtain $a(Y, Y) \geq\left(1-\tau_{2}(y)\right)\|Y\|_{\boldsymbol{V}}^{2}$ for all $Y \in \boldsymbol{V}$. Lax-Milgram lemma leads to the existence and uniqueness of $Y_{1}$ provided $\tau_{2}(y)<1$. Then, putting $w=Y_{1}$ in 2.11 implies

$$
a\left(Y_{1}, Y_{1}\right) \leq-\alpha \int_{\Omega} v \cdot Y_{1}-\nu \int_{\Omega} \nabla v \cdot \nabla Y_{1} \leq\left\|Y_{1}\right\|_{\boldsymbol{V}}\|v\|_{\boldsymbol{V}}=\left\|Y_{1}\right\|_{\boldsymbol{V}} \sqrt{2 E(y)}
$$

leading to 2.12). If $d=3$, using 2.4 , we obtain $a(Y, Y) \geq\left(1-\tau_{3}(y)\right)\|Y\|_{\boldsymbol{V}}^{2}$ for all $Y \in \boldsymbol{V}$ and we conclude as before.

We now check the differentiability of the least-squares functional.

Proposition 2.6. For all $y \in \boldsymbol{V}$, the map $Y \mapsto E(y+Y)$ is a differentiable function on the Hilbert space $\boldsymbol{V}$ and for any $Y \in \boldsymbol{V}$, we have

$$
E^{\prime}(y) \cdot Y=\int_{\Omega} \alpha v \cdot V+\nu \nabla v \cdot \nabla V
$$

where $V \in \boldsymbol{V}$ is the unique solution of

(2.15) $\alpha \int_{\Omega} V \cdot w+\nu \int_{\Omega} \nabla V \cdot \nabla w=-\alpha \int_{\Omega} Y \cdot w-\nu \int_{\Omega} \nabla Y \cdot \nabla w-\int_{\Omega}(y \cdot \nabla Y+Y \cdot \nabla y) \cdot w, \forall w \in \boldsymbol{V}$.

Proof. Let $y \in \boldsymbol{V}$ and $Y \in \boldsymbol{V}$. We have $E(y+Y)=\frac{1}{2}\|\bar{V}\|_{\boldsymbol{V}}^{2}$ where $\bar{V} \in \boldsymbol{V}$ is the unique solution of

$$
\begin{gathered}
\alpha \int_{\Omega} \bar{V} \cdot w+\nu \int_{\Omega} \nabla \bar{V} \cdot \nabla w+\alpha \int_{\Omega}(y+Y) \cdot w+\nu \int_{\Omega} \nabla(y+Y) \cdot \nabla w+\int_{\Omega}(y+Y) \cdot \nabla(y+Y) \cdot w \\
-\langle f, w\rangle_{\boldsymbol{V}^{\prime} \times \boldsymbol{V}}-\alpha \int_{\Omega} g w=0, \quad \forall w \in \boldsymbol{V} .
\end{gathered}
$$

If $v \in \boldsymbol{V}$ is the solution of (2.8) associated to $y, v^{\prime} \in \boldsymbol{V}$ is the unique solution of

$$
\alpha \int_{\Omega} v^{\prime} \cdot w+\nu \int_{\Omega} \nabla v^{\prime} \cdot \nabla w+\int_{\Omega} Y \cdot \nabla Y \cdot w=0, \quad \forall w \in \boldsymbol{V}
$$

and $V \in \boldsymbol{V}$ is the unique solution of 2.15, then it is straightforward to check that $\bar{V}-v-v^{\prime}-V \in$ $\boldsymbol{V}$ is solution of

$$
\alpha \int_{\Omega}\left(\bar{V}-v-v^{\prime}-V\right) \cdot w+\nu \int_{\Omega} \nabla\left(\bar{V}-v-v^{\prime}-V\right) \cdot \nabla w=0, \quad \forall w \in \boldsymbol{V}
$$

and therefore $\bar{V}-v-v^{\prime}-V=0$. Thus

$$
E(y+Y)=\frac{1}{2}\left\|v+v^{\prime}+V\right\|_{\boldsymbol{V}}^{2}=\frac{1}{2}\|v\|_{\boldsymbol{V}}^{2}+\frac{1}{2}\left\|v^{\prime}\right\|_{\boldsymbol{V}}^{2}+\frac{1}{2}\|V\|_{\boldsymbol{V}}^{2}+\left\langle V, v^{\prime}\right\rangle_{\boldsymbol{V}}+\langle V, v\rangle_{\boldsymbol{V}}+\left\langle v, v^{\prime}\right\rangle_{\boldsymbol{V}}
$$

Assume $d=2$. Then, writing (2.15) with $w=V$ and using 2.1), we obtain

$$
\begin{aligned}
\|V\|_{\boldsymbol{V}}^{2} & \leq\|V\|_{\boldsymbol{V}}\|Y\|_{\boldsymbol{V}}+\sqrt{2}\left(\|y\|_{2}\|\nabla Y\|_{2}+\|Y\|_{2}\|\nabla y\|_{2}\right)\|\nabla V\|_{2} \\
& \leq\|V\|_{\boldsymbol{V}}\|Y\|_{\boldsymbol{V}}+\frac{\sqrt{2}}{\sqrt{\alpha \nu}}\|y\|_{\boldsymbol{V}}\|Y\|_{\boldsymbol{V}}\|\nabla V\|_{2}
\end{aligned}
$$

leading to $\|V\|_{\boldsymbol{V}} \leq\|Y\|_{\boldsymbol{V}}\left(1+\frac{\sqrt{2}}{\sqrt{\alpha} \nu}\|y\|_{\boldsymbol{V}}\right)$. Similarly, using (2.16), we obtain $\left\|v^{\prime}\right\|_{\boldsymbol{V}} \leq \frac{1}{\sqrt{2 \alpha} \nu}\|Y\|_{\boldsymbol{V}}^{2}$. It follows that $\frac{1}{2}\left\|v^{\prime}\right\|_{\boldsymbol{V}}^{2}+\frac{1}{2}\|V\|_{\boldsymbol{V}}^{2}+\left\langle V, v^{\prime}\right\rangle_{\boldsymbol{V}}+\left\langle v, v^{\prime}\right\rangle_{\boldsymbol{V}}=o\left(\|Y\|_{\boldsymbol{V}}\right)$ and from 2.17 that

$$
E(y+Y)=E(y)+\langle v, V\rangle+o\left(\|Y\|_{\boldsymbol{V}}\right) .
$$

Eventually, the estimate $\left|\langle v, V\rangle_{\boldsymbol{V}}\right| \leq\|v\|_{\boldsymbol{V}}\|V\|_{\boldsymbol{V}} \leq\left(1+\frac{\sqrt{2}}{\sqrt{\alpha}}\|y\|_{\boldsymbol{V}}\right) \sqrt{E(y)}\|Y\|_{\boldsymbol{V}}$ gives the continuity of the linear map $Y \mapsto\langle v, V\rangle_{\boldsymbol{V}}$. The case $d=3$ is similar.

We are now in position to prove the following result which indicates that, in the ball $\left\{\tau_{d}(y)<\right.$ 1 ) of $\boldsymbol{V}$, any critical point for $E$ is also a zero of $E$. 
Proposition 2.7. For all $y \in \boldsymbol{V}$ satisfying $\tau_{d}(y)<1$,

$$
\left(1-\tau_{d}(y)\right) \sqrt{2 E(y)} \leq \frac{1}{\sqrt{\nu}}\left\|E^{\prime}(y)\right\|_{\boldsymbol{V}^{\prime}}
$$

Proof. For any $Y \in \boldsymbol{V}, E^{\prime}(y) \cdot Y=\int_{\Omega} \alpha v \cdot V+\nu \nabla v \cdot \nabla V$ where $V \in \boldsymbol{V}$ is the unique solution of 2.15. In particular, taking $Y=Y_{1}$ defined by (2.11), we obtain an element $V_{1} \in \boldsymbol{V}$ solution of

$$
\alpha \int_{\Omega} V_{1} \cdot w+\nu \int_{\Omega} \nabla V_{1} \cdot \nabla w=-\alpha \int_{\Omega} Y_{1} \cdot w-\nu \int_{\Omega} \nabla Y_{1} \cdot \nabla w-\int_{\Omega}\left(y \cdot \nabla Y_{1}+Y_{1} \cdot \nabla y\right) \cdot w, \forall w \in \boldsymbol{V}
$$

Summing 2.11 and 2.18, we obtain that $v-V_{1} \in \boldsymbol{V}$ solves

$$
\alpha \int_{\Omega}\left(v-V_{1}\right) \cdot w+\nu \int_{\Omega}\left(\nabla v-\nabla V_{1}\right) \cdot w=0, \quad \forall w \in \boldsymbol{V} .
$$

This implies that $v$ and $V_{1}$ coincide and then that

$$
E^{\prime}(y) \cdot Y_{1}=\int_{\Omega} \alpha|v|^{2}+\nu|\nabla v|^{2}=2 E(y), \quad \forall y \in \boldsymbol{V} .
$$

It follows that $2 E(y)=E^{\prime}(y) \cdot Y_{1} \leq\left\|E^{\prime}(y)\right\|_{\boldsymbol{V}^{\prime}}\left\|Y_{1}\right\|_{\boldsymbol{V}} \leq\left\|E^{\prime}(y)\right\|_{\boldsymbol{V}^{\prime}} \frac{\left\|Y_{1}\right\|_{\boldsymbol{V}}}{\sqrt{\nu}}$. Proposition 2.5 allows to conclude.

Eventually, we prove the following coercivity type inequality for the error functional $E$.

Proposition 2.8. Assume that a solution $\bar{y} \in \boldsymbol{V}$ of 2.5 satisfies $\tau_{d}(\bar{y})<1$. Then, for all $y \in \boldsymbol{V}$

$$
\|y-\bar{y}\|_{\boldsymbol{V}} \leq\left(1-\tau_{d}(\bar{y})\right)^{-1} \sqrt{2 E(y)} .
$$

Proof. For any $y \in \boldsymbol{V}$, let $v$ be the corresponding corrector and let $Y=y-\bar{y}$. We have

$\alpha \int_{\Omega} Y \cdot w+\nu \int_{\Omega} \nabla Y \cdot \nabla w+\int_{\Omega} y \cdot \nabla Y \cdot w+\int_{\Omega} Y \cdot \nabla \bar{y} \cdot w=-\alpha \int_{\Omega} v \cdot w-\nu \int_{\Omega} \nabla v \cdot \nabla w, \quad \forall w \in \boldsymbol{V}$.

For $w=Y$, this equality rewrites

$$
\|Y\|_{\boldsymbol{V}}^{2}=-\int_{\Omega} Y \cdot \nabla \bar{y} \cdot Y-\alpha \int_{\Omega} v \cdot Y-\nu \int_{\Omega} \nabla v \cdot \nabla Y .
$$

Repeating the arguments of the proof of Proposition 2.5, the result follows.

Assuming the existence of a solution of 2.5 in the ball $\left\{y \in \boldsymbol{V}, \tau_{d}(y)<1\right\}$, Proposition 2.7 and Proposition 2.8 imply that any minimizing sequence $\left\{y_{k}\right\}_{(k \in \mathbb{N})}$ for $E$ uniformly in $\{y \in$ $\left.\boldsymbol{V}, \tau_{d}(y) \leq 1\right\}$ strongly converges to a solution of 2.5 . Remark that, from Lemma 2.4. such solution is unique. In the next section, we construct, assuming the parameter $\alpha$ large enough, such sequence $\left\{y_{k}\right\}_{(k \in \mathbb{N})}$.

Remark 2.9. In order to simplify notations, we have introduced the corrector variable $v$ leading to the functional $E$. Instead, we may consider the functional $\widetilde{E}: \boldsymbol{V} \rightarrow \mathbb{R}$ defined by

$$
\widetilde{E}(y):=\frac{1}{2}\left\|\alpha y+\nu B_{1}(y)+B(y, y)-f+\alpha g\right\|_{\boldsymbol{V}^{\prime}}^{2}
$$

with $B_{1}: \boldsymbol{V} \rightarrow L^{2}(\Omega)^{d}$ and $B: \boldsymbol{V} \times \boldsymbol{V} \rightarrow L^{2}(\Omega)^{d}$ defined by $\left(B_{1}(y), w\right):=(\nabla y, \nabla w)_{2}$ and $(B(y, z), w):=\int_{\Omega} y \cdot \nabla z \cdot w$ respectively. $E$ and $\widetilde{E}$ are equivalent. Precisely, from the definition of $v$ (see (2.8)), we deduce that

$$
E(y)=\frac{1}{2}\|v\|_{\boldsymbol{V}}^{2} \leq \frac{c_{0}^{2}}{2 \nu^{2}}\left\|\alpha y+\nu B_{1}(y)+B(y, y)-f+\alpha g\right\|_{\boldsymbol{V}^{\prime}}^{2}=\frac{c_{0}^{2}}{\nu^{2}} \widetilde{E}(y), \quad \forall y \in \boldsymbol{V}
$$


Conversely,

$$
\begin{aligned}
\left\|\alpha y+\nu B_{1}(y)+B(y, y)-f+\alpha g\right\|_{\boldsymbol{V}^{\prime}} & =\sup _{w \in \boldsymbol{V}, w \neq 0} \frac{\int_{\Omega}(\alpha v \cdot w+\nu \nabla v \cdot \nabla w)}{\|w\|_{\boldsymbol{V}}} \\
& \leq\|v\|_{\boldsymbol{V}} \sup _{w \in \boldsymbol{V}, w \neq 0} \frac{\|w\|_{\boldsymbol{V}}}{\|w\|_{\boldsymbol{V}}} \leq \sqrt{\alpha c_{0}^{2}+\nu}\|v\|_{\boldsymbol{V}}
\end{aligned}
$$

so that $\widetilde{E}(y) \leq\left(\alpha c_{0}^{2}+\nu\right) E(y)$ for all $y \in \boldsymbol{V}$.

2.2. A strongly convergent minimizing sequence for $E$. We define in this section a sequence converging strongly to a solution of 2.5 for which $E$ vanishes. According to Proposition 2.7 , it suffices to define a minimizing sequence for $E$ included in the ball $\mathbb{B}=\left\{y \in \boldsymbol{V}, \tau_{d}(y)<1\right\}$. In this respect, remark that equality $(2.19)$ shows that $-Y_{1}$ given by the solution of (2.11) is a descent direction for the functional $E$. Therefore, we can define at least formally, for any $m \geq 1$, a minimizing sequence $\left\{y_{k}\right\}_{(k \geq 0)}$ as follows:

$$
\left\{\begin{array}{l}
y_{0} \in \boldsymbol{H} \quad \text { given, } \\
y_{k+1}=y_{k}-\lambda_{k} Y_{1, k}, \quad k \geq 0, \\
\lambda_{k}=\operatorname{argmin}_{\lambda \in[0, m]} E\left(y_{k}-\lambda Y_{1, k}\right)
\end{array}\right.
$$

with $Y_{1, k} \in \boldsymbol{V}$ the solution of the formulation

$\alpha \int_{\Omega} Y_{1, k} \cdot w+\nu \int_{\Omega} \nabla Y_{1, k} \cdot \nabla w+\int_{\Omega}\left(y_{k} \cdot \nabla Y_{1, k}+Y_{1, k} \cdot \nabla y_{k}\right) \cdot w=-\alpha \int_{\Omega} v_{k} \cdot w-\nu \int_{\Omega} \nabla v_{k} \cdot \nabla w, \forall w \in \boldsymbol{V}$ and $v_{k} \in \boldsymbol{V}$ the corrector (associated to $y_{k}$ ) solution of 2.8 leading (see $(2.19)$ ) to $E^{\prime}\left(y_{k}\right) \cdot Y_{1, k}=$ $2 E\left(y_{k}\right)$.

Remark that from 2.10, , the sequence $\left\{y_{k}\right\}_{k>0}$ is uniformly bounded since $y_{k}$ satisfies $\left\|y_{k}\right\|_{\boldsymbol{V}} \leq$ $\sqrt{2 E\left(y_{k}\right)}+\sqrt{\frac{c_{0}}{\nu}\|f\|_{H^{-1}}^{2}+\alpha\|g\|_{2}^{2}}$. However, we insist that, in order to justify the existence of the element $Y_{1, k}, y_{k}$ should satisfy $\tau_{d}\left(y_{k}\right)<1$, i.e. $\left\|\nabla y_{k}\right\|_{2}<\sqrt{2 \alpha \nu}$. We proceed in two steps: first, assuming that the sequence $\left\{y_{k}\right\}_{(k>0)}$ defined by 2.22 satisfies $\tau_{d}\left(y_{k}\right) \leq c_{1}<1$ for any $k$, we show that $E\left(y_{k}\right) \rightarrow 0$ and that $\left\{y_{k}\right\}$ converges strongly in $\boldsymbol{V}$ to a solution of (2.5). Then, we determine sufficient conditions on the initial guess $y_{0} \in \boldsymbol{V}$ in order that $\tau_{d}\left(y_{k}\right)<1$ for all $k \in \mathbb{N}$.

We start with the following lemma which provides the main property of the sequence $\left\{E\left(y_{k}\right)\right\}_{(k \geq 0)}$.

Lemma 2.10. Assume that the sequence $\left\{y_{k}\right\}_{(k \geq 0)}$ defined by (2.22) satisfies $\tau_{d}\left(y_{k}\right)<1$ for all $k \geq 0$. Then, for all $\lambda \in \mathbb{R}$, the following estimate holds true

$$
E\left(y_{k}-\lambda Y_{1, k}\right) \leq E\left(y_{k}\right)\left(|1-\lambda|+\lambda^{2} \frac{\left(1-\tau_{2}\left(y_{k}\right)\right)^{-2}}{\sqrt{\alpha} \nu} \sqrt{E\left(y_{k}\right)}\right)^{2}
$$

if $d=2$ and

$$
E\left(y_{k}-\lambda Y_{1, k}\right) \leq E\left(y_{k}\right)\left(|1-\lambda|+\lambda^{2} \frac{\sqrt{2}}{\sqrt{\nu}} \frac{M}{\left(\alpha \nu^{3}\right)^{1 / 4}}\left(1-\tau_{3}\left(y_{k}\right)\right)^{-2} \sqrt{E\left(y_{k}\right)}\right)^{2}
$$

if $d=3$.

Proof. For any real $\lambda$ and any $y_{k}, w_{k} \in \boldsymbol{V}$ we get the following expansion :

$$
\begin{aligned}
E\left(y_{k}-\lambda w_{k}\right)=E\left(y_{k}\right) & -\lambda \int_{\Omega}\left(\alpha v_{k} \cdot \bar{v}_{k}+\nu \nabla v_{k} \cdot \nabla \bar{v}_{k}\right) \\
& +\frac{\lambda^{2}}{2} \int_{\Omega}\left(\alpha\left|\bar{v}_{k}\right|^{2}+\nu\left|\nabla \bar{v}_{k}\right|^{2}+2\left(\alpha v_{k} \cdot \overline{\bar{v}}_{k}+\nu \nabla v_{k} \cdot \nabla \overline{\bar{v}}_{k}\right)\right) \\
& -\lambda^{3} \int_{\Omega} \alpha \bar{v}_{k} \overline{\bar{v}}_{k}+\nu \nabla \bar{v}_{k} \cdot \nabla \overline{\bar{v}}_{k}+\frac{\lambda^{4}}{2} \int_{\Omega} \alpha\left|\overline{\bar{v}}_{k}\right|^{2}+\nu\left|\nabla \overline{\bar{v}}_{k}\right|^{2}
\end{aligned}
$$


where $v_{k}, \bar{v}_{k} \in \boldsymbol{V}$ and $\overline{\bar{v}}_{k} \in \boldsymbol{V}$ solves respectively

$$
\begin{aligned}
& \alpha \int_{\Omega} v_{k} \cdot w+\nu \int_{\Omega} \nabla v_{k} \cdot \nabla w+\alpha \int_{\Omega} y_{k} \cdot w+\nu \int_{\Omega} \nabla y_{k} \cdot \nabla w+\int_{\Omega} y_{k} \cdot \nabla y_{k} \cdot w \\
&=<f, w>_{H^{-1}(\Omega)^{d} \times H_{0}^{1}(\Omega)^{d}}+\alpha \int_{\Omega} g \cdot w, \quad \forall w \in \boldsymbol{V}
\end{aligned}
$$

$\alpha \int_{\Omega} \bar{v}_{k} \cdot w+\nu \int_{\Omega} \nabla \bar{v}_{k} \cdot \nabla w+\alpha \int_{\Omega} w_{k} \cdot w+\nu \int_{\Omega} \nabla w_{k} \cdot \nabla w+\int_{\Omega} w_{k} \cdot \nabla y_{k} \cdot w+y_{k} \cdot \nabla w_{k} \cdot w=0, \quad \forall w \in \boldsymbol{V}$, and

$$
\alpha \int_{\Omega} \overline{\bar{v}}_{k} \cdot w+\nu \int_{\Omega} \nabla \overline{\bar{v}}_{k} \cdot \nabla w+\int_{\Omega} w_{k} \cdot \nabla w_{k} \cdot w=0, \quad \forall w \in \boldsymbol{V} .
$$

Since the corrector $\bar{v}_{k}$ associated to $Y_{1, k}$ coincides with the corrector $v_{k}$ associated to $y_{k}$, expansion 2.26) reduces to

$$
\begin{aligned}
E\left(y_{k}-\lambda Y_{1, k}\right) & =(1-\lambda)^{2} E\left(y_{k}\right)+\lambda^{2}(1-\lambda) \int_{\Omega} \alpha v_{k} \cdot \overline{\bar{v}}_{k}+\nu \nabla v_{k} \cdot \nabla \overline{\bar{v}}_{k}+\frac{\lambda^{4}}{2} \int_{\Omega} \alpha\left|\overline{\bar{v}}_{k}\right|^{2}+\nu\left|\nabla \overline{\bar{v}}_{k}\right|^{2} \\
& \leq(1-\lambda)^{2} E\left(y_{k}\right)+\lambda^{2}(1-\lambda)\left\|v_{k}\right\|\left\|_ { \boldsymbol { V } } \left|\left\|\overline{\bar{v}}_{k}\left|\left\|_{\boldsymbol{V}}+\frac{\lambda^{4}}{2}\right\|\right| \overline{\bar{v}}_{k} \mid\right\|_{\boldsymbol{V}}^{2}\right.\right. \\
& \leq\left(|1-\lambda| \sqrt{E\left(y_{k}\right)}+\frac{\lambda^{2}}{\sqrt{2}}\left\|\overline{\bar{v}}_{k} \mid\right\|_{\boldsymbol{V}}\right)^{2} .
\end{aligned}
$$

Moreover, if $d=2$, then 2.29 leads to $\left\|\overline{\bar{v}}_{k}\right\|_{\boldsymbol{V}} \leq \frac{\left\|Y_{1, k}\right\|_{V}^{2}}{\sqrt{2 \alpha} \nu} \leq \sqrt{2}\left(1-\tau_{2}\left(y_{k}\right)\right)^{-2} \frac{E\left(y_{k}\right)}{\sqrt{\alpha} \nu}$ and then to (2.24). If $d=3$, then

$$
\left\|\overline{\bar{v}}_{k}\right\|_{\boldsymbol{V}} \leq \frac{1}{\sqrt{\nu}} \frac{M}{\left(\alpha \nu^{3}\right)^{1 / 4}}\left\|Y_{1, k}\right\|_{\boldsymbol{V}}^{2} \leq \frac{1}{\sqrt{\nu}} \frac{M}{\left(\alpha \nu^{3}\right)^{1 / 4}} 2\left(1-\tau_{3}\left(y_{k}\right)\right)^{-2} E\left(y_{k}\right)
$$

leading to 2.25 .

We are now in position to prove the following convergence result for the sequence $\left\{E\left(y_{k}\right)\right\}_{(k \geq 0)}$.

Proposition 2.11. Let $\left\{y_{k}\right\}_{k \geq 0}$ be the sequence defined by 2.22). Assume that there exists a constant $c_{1} \in(0,1)$ such that $\tau_{d}\left(y_{k}\right) \leq c_{1}$ for all $k$. Then $E\left(y_{k}\right) \rightarrow 0$ as $k \rightarrow \infty$. Moreover, there exists $k_{0} \in \mathbb{N}$ such that the sequence $\left\{E\left(y_{k}\right)\right\}_{\left(k \geq k_{0}\right)}$ decays quadratically.

Proof. Consider the case $d=2$. The inequality $\tau_{2}\left(y_{k}\right) \leq c_{1}$ and 2.24 implies that

$$
E\left(y_{k}-\lambda Y_{1, k}\right) \leq E\left(y_{k}\right)\left(|1-\lambda|+\lambda^{2} c_{\alpha, \nu} \sqrt{E\left(y_{k}\right)}\right)^{2}, \quad c_{\alpha, \nu}:=\frac{\left(1-c_{1}\right)^{-2}}{\sqrt{\alpha} \nu}
$$

Let us denote the polynomial $p_{k}$ by $p_{k}(\lambda)=|1-\lambda|+\lambda^{2} c_{\alpha, \nu} \sqrt{E\left(y_{k}\right)}$ for all $\lambda \in[0, m]$. So, we can write

$$
\sqrt{E\left(y_{k+1}\right)}=\min _{\lambda \in[0, m]} \sqrt{E\left(y_{k}-\lambda Y_{1, k}\right)} \leq \min _{\lambda \in[0, m]} p_{k}(\lambda) \sqrt{E\left(y_{k}\right)} .
$$

If $c_{\alpha, \nu} \sqrt{E\left(y_{0}\right)}<1$ (and thus $c_{\alpha, \nu} \sqrt{E\left(y_{k}\right)}<1$ for all $k \in \mathbb{N}$ ) then

$$
p_{k}\left(\widetilde{\lambda}_{k}\right):=\min _{\lambda \in[0, m]} p_{k}(\lambda) \leq p_{k}(1)=c_{\alpha, \nu} \sqrt{E\left(y_{k}\right)}
$$

and thus

$$
c_{\alpha, \nu} \sqrt{E\left(y_{k+1}\right)} \leq\left(c_{\alpha, \nu} \sqrt{E\left(y_{k}\right)}\right)^{2}
$$

implying that $c_{\alpha, \nu} \sqrt{E\left(y_{k}\right)} \rightarrow 0$ as $k \rightarrow \infty$ with a quadratic rate. 
Suppose now that $c_{\alpha, \nu} \sqrt{E\left(y_{0}\right)} \geq 1$ and denote $I=\left\{k \in \mathbb{N}, c_{\alpha, \nu} \sqrt{E\left(y_{k}\right)} \geq 1\right\}$. Let us prove that $I$ is a finite subset of $\mathbb{N}$. For all $k \in I$, since $c_{\alpha, \nu} \sqrt{E\left(y_{k}\right)} \geq 1$,

$$
\min _{\lambda \in[0, m]} p_{k}(\lambda)=\min _{\lambda \in[0,1]} p_{k}(\lambda)=p_{k}\left(\frac{1}{2 c_{\alpha, \nu} \sqrt{E\left(y_{k}\right)}}\right)=1-\frac{1}{4 c_{\alpha, \nu} \sqrt{E\left(y_{k}\right)}}
$$

and thus, for all $k \in I$,

$$
c_{\alpha, \nu} \sqrt{E\left(y_{k+1}\right)} \leq\left(1-\frac{1}{4 c_{\alpha, \nu} \sqrt{E\left(y_{k}\right)}}\right) c_{\alpha, \nu} \sqrt{E\left(y_{k}\right)}=c_{\alpha, \nu} \sqrt{E\left(y_{k}\right)}-\frac{1}{4} .
$$

This inequality implies that the sequence $\left\{c_{\alpha, \nu} \sqrt{E\left(y_{k}\right)}\right\}_{k \in \mathbb{N}}$ strictly decreases and thus, there exists $k_{0} \in \mathbb{N}$ such that for all $k \geq k_{0}, c_{\alpha, \nu} \sqrt{E\left(y_{k}\right)}<1$. Thus $I$ is a finite subset of $\mathbb{N}$. Arguing as in the first case, it follows that $c_{\alpha, \nu} \sqrt{E\left(y_{k}\right)} \rightarrow 0$ as $k \rightarrow \infty$.

In both cases, remark that $p_{k}\left(\widetilde{\lambda}_{k}\right)$ decreases with respect to $k$. The case $d=3$ is similar with

$$
c_{\alpha, \nu}=\frac{\sqrt{2}}{\sqrt{\nu}} \frac{M}{\left(\alpha \nu^{3}\right)^{1 / 4}}\left(1-c_{1}\right)^{-2} .
$$

Lemma 2.12. Assume that the sequence $\left\{y_{k}\right\}_{(k \geq 0)}$ defined by 2.22) satisfies $\tau_{d}\left(y_{k}\right) \leq c_{1}$ for all $k$ and some $c_{1} \in(0,1)$. Then $\lambda_{k} \rightarrow 1$ as $k \rightarrow \infty$.

Proof. In view of 2.30, we have, as long as $E\left(y_{k}\right)>0$,

$$
\left(1-\lambda_{k}\right)^{2}=\frac{E\left(y_{k+1}\right)}{E\left(y_{k}\right)}-\lambda_{k}^{2}\left(1-\lambda_{k}\right) \frac{\left\langle v_{k}, \overline{\bar{v}}_{k}\right\rangle_{\boldsymbol{V}}}{E\left(y_{k}\right)}-\lambda_{k}^{4} \frac{\left\|\overline{\bar{v}}_{k}\right\| \|_{\boldsymbol{V}}^{2}}{2 E\left(y_{k}\right)} .
$$

From the proof of Lemma 2.10 $\frac{\left\langle v_{k}, \overline{\bar{v}}_{k}\right\rangle_{V}}{E\left(y_{k}\right)} \leq C(\alpha, \nu)\left(1-c_{1}\right)^{-2} \sqrt{E\left(y_{k}\right)}$ while $\frac{\left\|\overline{\bar{v}}_{k}\right\|_{V}^{2}}{E\left(y_{k}\right)} \leq C(\alpha, \nu)^{2}(1-$ $\left.c_{1}\right)^{-4} E\left(y_{k}\right)$. Consequently, since $\lambda_{k} \in[0, m]$ and $\frac{E\left(y_{k+1}\right)}{E\left(y_{k}\right)} \rightarrow 0$, we deduce that $\left(1-\lambda_{k}\right)^{2} \rightarrow 0$, that is $\lambda_{k} \rightarrow 1$ as $k \rightarrow \infty$.

Proposition 2.13. Let $\left\{y_{k}\right\}_{(k \geq 0)}$ be the sequence defined by 2.22). Assume that there exists a constant $c_{1} \in(0,1)$ such that $\tau_{d}\left(y_{k}\right) \leq c_{1}$ for all $k$. Then, $y_{k} \rightarrow \bar{y}$ in $\boldsymbol{V}$ where $\bar{y} \in \boldsymbol{V}$ is the unique solution of 2.5 .

Proof. Remark that we can not use Proposition 2.8 since we do not know yet that there exists a solution, say $z$, of 2.5 satisfying $\tau_{d}(z)<1$. In view of $y_{k+1}=y_{0}-\sum_{n=0}^{k} \lambda_{n} Y_{1, n}$, we write

$$
\sum_{n=0}^{k}\left|\lambda_{n}\right|\left|\left\|Y_{1, n}\right\|_{\boldsymbol{V}} \leq m \sum_{n=0}^{k}\left\|\mid Y_{1, n}\right\|_{\boldsymbol{V}} \leq m \sqrt{2} \sum_{n=0}^{k} \frac{\sqrt{E\left(y_{n}\right)}}{1-\tau_{d}\left(y_{n}\right)} \leq \frac{m \sqrt{2}}{1-c_{1}} \sum_{n=0}^{k} \sqrt{E\left(y_{n}\right)} .\right.
$$

Using that $p_{n}\left(\widetilde{\lambda}_{n}\right) \leq p_{0}\left(\widetilde{\lambda}_{0}\right)$ for all $n \geq 0$, we can write for $n>0$,

$$
\sqrt{E\left(y_{n}\right)} \leq p_{n-1}\left(\widetilde{\lambda}_{n-1}\right) \sqrt{E\left(y_{n-1}\right)} \leq p_{0}\left(\widetilde{\lambda}_{0}\right) \sqrt{E\left(y_{n-1}\right)} \leq p_{0}\left(\widetilde{\lambda}_{0}\right)^{n} \sqrt{E\left(y_{0}\right)},
$$

and we finally obtain

$$
\sum_{n=0}^{k}\left|\lambda_{n}\right||| \mid Y_{1, n} \|_{\boldsymbol{V}} \leq \frac{m \sqrt{2}}{1-c_{1}} \frac{\sqrt{E\left(y_{0}\right)}}{1-p_{0}\left(\widetilde{\lambda}_{0}\right)}
$$

for which we deduce that the serie $\sum_{k \geq 0} \lambda_{k} Y_{1, k}$ converges in $\boldsymbol{V}$. Then, $y_{k}$ converges in $\boldsymbol{V}$ to $\bar{y}:=y_{0}+\sum_{k \geq 0} \lambda_{k} Y_{1, k}$. Eventually, the convergence of $E\left(y_{k}\right)$ to 0 implies the convergence of the corrector $v_{k}$ to 0 in $\boldsymbol{V}$; taking the limit in the corrector equation (2.27) shows that $\bar{y}$ solves 2.5. Since $\tau_{d}(\bar{y}) \leq c_{1}<1$, Lemma 2.4 shows that this solution is unique.

As mentioned earlier, the remaining and crucial point is to show that the sequence $\left\{y_{k}\right\}_{(k \geq 0)}$ satisfies the uniform property $\tau_{d}\left(y_{k}\right) \leq c_{1}$ for some $c_{1}<1$. 
Lemma 2.14. Assume that $y_{0}=g \in \boldsymbol{V}$. For all $c_{1} \in(0,1)$ there exists $\alpha_{0}>0$, such that, for any $\alpha \geq \alpha_{0}$, the unique sequence defined by 2.22 satisfies $\tau_{d}\left(y_{k}\right) \leq c_{1}$ for all $k \geq 0$.

Proof. Let $c_{1} \in(0,1)$ and assume that $y_{0}$ belongs to $\boldsymbol{V}$. There exists $\alpha_{1}>0$ such that for all $\alpha \geq \alpha_{1} \tau_{d}\left(y_{0}\right) \leq \frac{c_{1}}{2}$.

Moreover, in view of the above computation, for all $\alpha>0$, since for all $v \in \boldsymbol{V}\|v\|_{\boldsymbol{V}} \leq \frac{1}{\nu}\|v\|_{\boldsymbol{V}}$, for all $k \in \mathbb{N}$

where

$$
\left\|y_{k+1}\right\|_{\boldsymbol{V}} \leq\left\|y_{0}\right\|_{\boldsymbol{V}}+\frac{m \sqrt{2}}{\nu\left(1-c_{1}\right)} \frac{\sqrt{E\left(y_{0}\right)}}{1-p_{0}\left(\widetilde{\lambda}_{0}\right)}
$$

$$
\frac{\sqrt{E\left(y_{0}\right)}}{1-p_{0}\left(\widetilde{\lambda}_{0}\right)} \leq\left\{\begin{array}{lll}
\frac{\sqrt{E\left(y_{0}\right)}}{1-c_{\alpha, \nu} \sqrt{E\left(y_{0}\right)}}, & \text { if } & c_{\alpha, \nu} \sqrt{E\left(y_{0}\right)}<1, \\
4 c_{\alpha, \nu} E\left(y_{0}\right), & \text { if } \quad & c_{\alpha, \nu} \sqrt{E\left(y_{0}\right)} \geq 1 .
\end{array}\right.
$$

Assume $d=2$. From 2.8), we obtain that

$$
\|v\|_{\boldsymbol{V}}^{2} \leq \alpha\|g-y\|_{2}^{2}+\frac{1}{\nu}\left(\nu\|\nabla y\|_{2}+\|y\|_{2}\|\nabla y\|_{2}+\sqrt{c_{0}}\|f\|_{H^{-1}(\Omega)^{2}}\right)^{2} .
$$

In particular, taking $y=y_{0}=g$ allows to remove the $\alpha$ term in the right hand side and gives

$$
E(g) \leq \frac{1}{2 \nu}\left(\|g\|_{\boldsymbol{V}}\left(\nu+\|g\|_{2}\right)+\sqrt{c_{0}}\|f\|_{H^{-1}(\Omega)^{2}}\right)^{2}:=\frac{1}{2 \nu} c_{2}(f, g),
$$

and thus, if $c_{\alpha_{1}, \nu} \sqrt{E(g)} \geq 1$, then for all $\alpha \geq \alpha_{1}$ such that $c_{\alpha, \nu} \sqrt{E(g)} \geq 1$ and for all $k \in \mathbb{N}$ :

$$
\left\|y_{k+1}\right\|_{\boldsymbol{V}} \leq\|g\|_{\boldsymbol{V}}+\frac{m \sqrt{2}}{\nu\left(1-c_{1}\right)} \frac{\sqrt{E(g)}}{1-p_{0}\left(\widetilde{\lambda}_{0}\right)} \leq\|g\|_{\boldsymbol{V}}+\frac{2 m \sqrt{2}}{\nu^{3} \sqrt{\alpha}\left(1-c_{1}\right)^{3}} c_{2}(f, g) .
$$

If now $c_{\alpha_{1}, \nu} \sqrt{E(g)}<1$ then there exists $0<K<1$ such that for all $\alpha \geq \alpha_{1}$ we have $c_{\alpha, \nu} \sqrt{E(g)} \leq K$. We therefore have for all $\alpha \geq \alpha_{1}$

$$
\frac{\sqrt{E(g)}}{1-p_{0}\left(\widetilde{\lambda}_{0}\right)} \leq \frac{\sqrt{E(g)}}{1-K}
$$

and thus for all $k \in \mathbb{N}$ :

$$
\left\|y_{k+1}\right\|_{\boldsymbol{V}} \leq\|g\|_{\boldsymbol{V}}+\frac{m \sqrt{2}}{\nu\left(1-c_{1}\right)} \frac{\sqrt{E(g)}}{1-p_{0}\left(\widetilde{\lambda}_{0}\right)} \leq\|g\|_{\boldsymbol{V}}+\frac{m}{\nu^{3 / 2}\left(1-c_{1}\right)(1-K)} \sqrt{c_{2}(f, g)} .
$$

On the other hand, there exists $\alpha_{0} \geq \alpha_{1}$ such that, for all $\alpha \geq \alpha_{0}$ we have

$$
\frac{2 m \sqrt{2}}{\nu^{3} \sqrt{\alpha}\left(1-c_{1}\right)^{3}} c_{2}(f, g) \leq \frac{c_{1}}{2} \sqrt{2 \alpha \nu}
$$

and

$$
\frac{m}{\nu^{3 / 2}\left(1-c_{1}\right)(1-K)} \sqrt{c_{2}(f, g)} \leq \frac{c_{1}}{2} \sqrt{2 \alpha \nu}
$$

We then deduce from 2.34 and 2.35) that for all $\alpha \geq \alpha_{0}$ and for all $k \in \mathbb{N}$ :

$$
\left\|y_{k+1}\right\|_{V} \leq \frac{c_{1}}{2} \sqrt{2 \alpha \nu}+\frac{c_{1}}{2} \sqrt{2 \alpha \nu}=c_{1} \sqrt{2 \alpha \nu}
$$

that is $\tau_{2}\left(y_{k+1}\right) \leq c_{1}$.

Assume $d=3$. We argue as in the case $d=2$ and deduce from 2.8), since $y_{0}=g$, that

$$
E(g) \leq \frac{1}{2 \nu}\left(\|g\|_{\boldsymbol{V}}\left(\nu+c\left\|y_{0}\right\|_{2}^{1 / 2}\|g\|_{\boldsymbol{V}}^{1 / 2}\right)+\sqrt{c_{0}}\|f\|_{H^{-1}(\Omega)^{3}}\right)^{2}:=\frac{1}{2 \nu} c_{3}(f, g)
$$

and thus, if $c_{\alpha_{1}, \nu} \sqrt{E(g)} \geq 1$, then for all $\alpha \geq \alpha_{1}$ such that $c_{\alpha, \nu} \sqrt{E(g)} \geq 1$ and for all $k \in \mathbb{N}$ :

$$
\left\|y_{k+1}\right\|_{\boldsymbol{V}} \leq\|g\|_{\boldsymbol{V}}+\frac{m \sqrt{2}}{\nu\left(1-c_{1}\right)} \frac{\sqrt{E(g)}}{1-p_{0}\left(\widetilde{\lambda}_{0}\right)} \leq\|g\|_{\boldsymbol{V}}+\frac{4 m M}{\nu^{5 / 2}\left(\alpha \nu^{3}\right)^{1 / 4}\left(1-c_{1}\right)^{3}} c_{3}(f, g) .
$$


If now $c_{\alpha_{1}, \nu} \sqrt{E(g)}<1$ then there exists $0<K<1$ such that for all $\alpha \geq \alpha_{1}$ we have $c_{\alpha, \nu} \sqrt{E(g)} \leq K$. We therefore have for all $\alpha \geq \alpha_{1}$

$$
\frac{\sqrt{E(g)}}{1-p_{0}\left(\widetilde{\lambda}_{0}\right)} \leq \frac{\sqrt{E\left(y_{0}\right)}}{1-K}
$$

and thus for all $k \in \mathbb{N}$ :

$$
\left\|y_{k+1}\right\|_{\boldsymbol{V}} \leq\|g\|_{\boldsymbol{V}}+\frac{m \sqrt{2}}{\nu\left(1-c_{1}\right)} \frac{\sqrt{E(g)}}{1-p_{0}\left(\widetilde{\lambda}_{0}\right)} \leq\|g\|_{\boldsymbol{V}}+\frac{m}{\nu^{3 / 2}\left(1-c_{1}\right)(1-K)} \sqrt{c_{3}(f, g)} .
$$

On the other hand, there exists $\alpha_{0} \geq \alpha_{1}$ such that, for all $\alpha \geq \alpha_{0}$ we have

and

$$
\frac{4 m M}{\nu^{5 / 2}\left(\alpha \nu^{3}\right)^{1 / 4}\left(1-c_{1}\right)^{3}} c_{3}(f, g) \leq \frac{c_{1}}{2} \frac{\left(\alpha \nu^{3}\right)^{1 / 4}}{M}
$$

$$
\frac{m}{\nu^{3 / 2}\left(1-c_{1}\right)(1-K)} \sqrt{c_{3}(f, g)} \leq \frac{c_{1}}{2} \frac{\left(\alpha \nu^{3}\right)^{1 / 4}}{M} .
$$

We then deduce from 2.37) and 2.38 that for all $\alpha \geq \alpha_{0}$ and for all $k \in \mathbb{N}$ :

$$
\left\|y_{k+1}\right\|_{\boldsymbol{V}} \leq \frac{c_{1}}{2} \frac{\left(\alpha \nu^{3}\right)^{1 / 4}}{M}+\frac{c_{1}}{2} \frac{\left(\alpha \nu^{3}\right)^{1 / 4}}{M}=c_{1} \frac{\left(\alpha \nu^{3}\right)^{1 / 4}}{M}
$$

that is $\tau_{3}\left(y_{k+1}\right) \leq c_{1}$.

Gathering the previous lemmas and propositions, we can now deduce the strong convergence of the sequence $\left\{y_{k}\right\}_{k \geq 0}$ defined by 2.22 , initialized by $y_{0}=g$.

Theorem 2.15. Let $c_{1} \in(0,1)$. Assume that $y_{0}=g \in \boldsymbol{V}$ and $\alpha$ is large enough so that

$$
\begin{aligned}
& c_{2}(f, g) \leq \max \left(\frac{1-c_{1}}{2}, \frac{c_{1} \sqrt{\nu}\left(1-K^{2}\right)}{m}\right) \frac{c_{1}}{4 m} \nu^{5 / 2}\left(1-c_{1}\right)^{2} 2 \alpha \nu, \quad \text { if } \quad d=2, \\
& c_{3}(f, g) \leq \max \left(\frac{1-c_{1}}{2}, \frac{c_{1} \sqrt{\nu}(1-K)^{2}}{m}\right) \frac{c_{1}}{4 m M^{2}} \nu^{5 / 2}\left(1-c_{1}\right)^{2}\left(\alpha \nu^{3}\right)^{1 / 2}, \quad \text { if } \quad d=3,
\end{aligned}
$$

where $c_{2}(f, g)$ and $c_{3}(f, g)$ are defined in (2.33) and (2.36) respectively. Then, the sequence $\left\{y_{k}\right\}_{(k \in \mathbb{N})}$ defined by 2.22 strongly converges to the unique solution y of (2.5). Moreover, there exists $k_{0} \in \mathbb{N}$ such that the sequence $\left\{y_{k}\right\}_{k \geq k_{0}}$ converges quadratically to $y$. Moreover, this solution satisfies $\tau_{d}(y)<1$.

2.3. Remarks. The following remarks are in order.

Remark 2.16. Estimate (2.6) is usually used to obtain a sufficient condition on the data $f, g$ to ensure the uniqueness of the solution of (2.5) (i.e. $\left.\tau_{d}(y)<1\right)$ : it leads to

$$
\begin{aligned}
& \alpha\|g\|_{2}^{2}+c_{0} \nu\|f\|_{\left(H^{-1}(\Omega)\right)^{2}}^{2} \leq 2 \alpha \nu^{2}, \quad \text { if } \quad d=2, \\
& \alpha\|g\|_{2}^{2}+c_{0} \nu\|f\|_{\left(H^{-1}(\Omega)\right)^{3}}^{2} \leq \frac{\nu\left(\alpha \nu^{3}\right)^{1 / 2}}{M^{2}}, \quad \text { if } \quad d=3 .
\end{aligned}
$$

We emphasize that such (sufficient) conditions are more restrictive than (2.39), as they impose smallness properties on $g$ : precisely $\|g\|_{2}^{2} \leq 2 \nu^{2}$ if $d=2$ and $\|g\|_{2}^{2} \leq \frac{\nu^{5 / 2}}{M^{2} \alpha^{1 / 2}}$ if $d=3$. In particular, this latter yields a restrictive condition for $\alpha$ large contrary to (2.39).

Remark 2.17. It seems surprising that the algorithm (2.22) achieves a quadratic rate for $k$ large. Let us consider the application $\mathcal{F}: \boldsymbol{V} \rightarrow \boldsymbol{V}^{\prime}$ defined as $\mathcal{F}(y)=\alpha y+\nu B_{1}(y)+B(y, y)-f-\alpha g$. The sequence $\left\{y_{k}\right\}_{(k>0)}$ associated to the Newton method to find the zero of $\mathcal{F}$ is defined as follows:

$$
\left\{\begin{array}{l}
y_{0} \in \boldsymbol{V} \\
\mathcal{F}^{\prime}\left(y_{k}\right) \cdot\left(y_{k+1}-y_{k}\right)=-\mathcal{F}\left(y_{k}\right), \quad k \geq 0 .
\end{array}\right.
$$


We check that this sequence coincides with the sequence obtained from (2.22) if $\lambda_{k}$ is fixed equal to one and if $y_{0} \in \boldsymbol{V}$. The algorithm (2.22) which consists to optimize the parameter $\lambda_{k} \in[0, m]$, $m \geq 1$, in order to minimize $E\left(y_{k}\right)$, equivalently $\left\|\mathcal{F}\left(y_{k}\right)\right\|_{\boldsymbol{V}^{\prime}}$, corresponds to the so-called in the literature damped Newton method for the application $\mathcal{F}$ (see [3]). As the iterates increase, the optimal parameter $\lambda_{k}$ converges to one (according to Lemma 2.12), this globally convergent method behaves like the standard Newton method (for which $\lambda_{k}$ is fixed equal to one): this explains the quadratic rate after a finite number of iterates. To the best of our knowledge, this is the first analysis of the damped Newton method for a partiel differential equation. Among the few numerical works devoted to the damped Newton method for partial differential equations, we mention [16] for computing viscoplastic fluid flows.

Remark 2.18. Section 6, chapter 6 of the book 4] introduces a least-squares method in order to solve an Oseen type equation (without incompressibility constraint). The convergence of any minimizing sequence toward a solution $y$ is proved under the a priori assumption that the operator $D F(y)$ defined as follows

$$
D F(y) \cdot w=\alpha w-\nu \Delta w+[(w \cdot \nabla) y+(y \cdot \nabla) w], \quad \forall w \in \boldsymbol{V}
$$

(for some $\alpha>0$ ) is an isomorphism from $\boldsymbol{V}$ onto $\boldsymbol{V}^{\prime} . y$ is then said to be a nonsingular point. According to Proposition 2.5, a sufficient condition for $y$ to be a nonsingular point is $\tau_{d}(y)<1$. Recall that $\tau_{d}$ depends on $\alpha$. As far as we know, determining a weaker condition ensuring that $\operatorname{DF}(y)$ is an isomorphism is an open question. Moreover, according to Lemma 2.4. it turns out that this condition is also a sufficient condition for the uniqueness of (2.5). Theorem 2.15 asserts that, if $\alpha$ is large enough, then the sequence $\left\{y_{k}\right\}_{(k \in \mathbb{N})}$ defined in 2.22 , initialized with $y_{0}=g$, is a convergent sequence of nonsingular points. Since $\lambda_{k}$ is constant equal to one, this shows the convergence of the Newton method to solve the steady Navier-Stokes equation.

Remark 2.19. We may also define a minimizing sequence for $E$ using the gradient $E^{\prime}$ :

$$
\left\{\begin{array}{l}
y_{0} \in \boldsymbol{H} \quad \text { given, } \\
y_{k+1}=y_{k}-\lambda_{k} g_{k}, \quad k \geq 0, \\
\lambda_{k}=\operatorname{argmin}_{\lambda \in[0, m]} E\left(y_{k}-\lambda g_{k}\right)
\end{array}\right.
$$

with $g_{k} \in \boldsymbol{V}$ such that $\left(g_{k}, w\right)_{\boldsymbol{V}}=\left(E^{\prime}\left(y_{k}\right), w\right)_{\boldsymbol{V}^{\prime}, \boldsymbol{V}}$ for all $w \in \boldsymbol{V}$. In particular, $\left\|g_{k}\right\|_{\boldsymbol{V}}=$ $\left\|E^{\prime}\left(y_{k}\right)\right\|_{V^{\prime}}$. Using the expansion 2.17) with $w_{k}=g_{k}$, we can prove the linear decrease of the sequence $\left\{E\left(y_{k}\right)\right\}_{k>0}$ to zero assuming however that $E\left(y_{0}\right)$ is small enough, of the order of $\nu^{2}$, independently of the value of $\alpha$ (we refer to [11, Lemma 4.1] in a similar context).

\section{Application to the BackWard Euler scheme}

We now use the results of the previous section to discuss the resolution of the backward Euler scheme (1.2) through a least-squares method. The weak formulation of this scheme reads as follows: given $y^{0}=u_{0} \in \boldsymbol{H}$, the sequence $\left\{y^{n}\right\}_{n>0}$ in $\boldsymbol{V}$ is defined by recurrence as follows:

$$
\int_{\Omega} \frac{y^{n+1}-y^{n}}{\delta t} \cdot w+\nu \int_{\Omega} \nabla y^{n+1} \cdot \nabla w+\int_{\Omega} y^{n+1} \cdot \nabla y^{n+1} \cdot w=<f^{n}, w>_{H^{-1}(\Omega)^{d} \times H_{0}^{1}(\Omega)^{d}}
$$

with $f^{n}$ defined by (1.4) in term of the external force of the Navier-Stokes model (1.1). We recall that a piecewise linear interpolation in time of $\left\{y^{n}\right\}_{n \geq 0}$ weakly converges in $L^{2}(0, T ; \boldsymbol{V})$ toward a solution of 1.1

As done in 2], one may use the least-squares method (analyzed in Section 2) to solve iteratively 3.1. Precisely, in order to approximate $y^{n+1}$ from $y^{n}$, one may consider the following extremal problem

$$
\inf _{y \in \boldsymbol{V}} E_{n}(y), \quad E_{n}(y)=\frac{1}{2}\|v\|_{\boldsymbol{V}}^{2}
$$


where the corrector $v \in \boldsymbol{V}$ solves

$$
\begin{aligned}
\alpha \int_{\Omega} v \cdot w+\nu \int_{\Omega} \nabla v \cdot \nabla w=-\alpha & \int_{\Omega} y \cdot w-\nu \int_{\Omega} \nabla y \cdot \nabla w-\int_{\Omega} y \cdot \nabla y \cdot w \\
& +<f^{n}, w>_{H^{-1}(\Omega)^{d} \times H_{0}^{1}(\Omega)^{d}}+\alpha \int_{\Omega} y^{n} \cdot w, \quad \forall w \in \boldsymbol{V}
\end{aligned}
$$

with $\alpha$ and $f^{n}$ given by 1.4 . For any $n \geq 0$, a minimizing sequence $\left\{y_{k}^{n}\right\}_{(k \geq 0)}$ for $E_{n}$ is defined as follows :

$$
\left\{\begin{array}{l}
y_{0}^{n+1}=y^{n}, \\
y_{k+1}^{n+1}=y_{k}^{n+1}-\lambda_{k} Y_{1, k}^{n+1}, \quad k \geq 0, \\
\lambda_{k}=\operatorname{argmin}_{\lambda \in[0, m]} E_{n}\left(y_{k}^{n+1}-\lambda Y_{1, k}^{n+1}\right)
\end{array}\right.
$$

where $Y_{1, k}^{n} \in \boldsymbol{V}$ solves 2.23). Remark that, in view of Theorem 2.15 the first element of the minimizing sequence is chosen equal to $y^{n}$, i.e. the minimizer of $E_{n-1}$.

The main goal of this section is to prove that for all $n \in \mathbb{N}$, the minimizing sequence $\left(y_{k}^{n+1}\right)_{k \in \mathbb{N}}$ do converges to a solution $y^{n+1}$ of (3.1). This allows to justify the use of least-squares method to solve the backward Euler scheme. Arguing as in Lemma 2.14, we have to prove the existence of a constant $c_{1} \in(0,1)$, such that $\tau_{d}\left(y_{k}^{n}\right) \leq c_{1}$ for all $n$ and $k$ in $\mathbb{N}$. Remark that the initialization $y_{0}^{n+1}$ is fixed as the minimizer of the functional $E^{n-1}$, obtained at the previous iterate. Consequently, the uniform property $\tau_{d}\left(y_{k}^{n}\right) \leq c_{1}$ is related to the initial guess $y_{0}^{0}$ equal to the initial position $u_{0}$, to the external force $F$ (see (1.1)) and to the value of $\alpha . u_{0}$ and $F$ are given a priori. On the other hand, the parameter $\alpha$, related to the discretization parameter $\delta t$, can be chosen as large as necessary. As we shall see, this uniform property, which is essential to set up the least-squares procedure, requires smallness properties on $u_{0}$ and $F$.

We start with the following result analogue to Proposition 2.3 .

Proposition 3.1. Let $\left(f^{n}\right)_{n \in \mathbb{N}}$ be a sequence in $H^{-1}(\Omega)^{d}, \alpha>0$ and $y^{0}=u_{0} \in \boldsymbol{H}$. For any $n \in \mathbb{N}$, there exists a solution $y^{n+1} \in \boldsymbol{V}$ of

(3.5) $\alpha \int_{\Omega}\left(y^{n+1}-y^{n}\right) \cdot w+\nu \int_{\Omega} \nabla y^{n+1} \cdot \nabla w+\int_{\Omega} y^{n+1} \cdot \nabla y^{n+1} \cdot w=<f^{n}, w>_{H^{-1}(\Omega)^{d} \times H_{0}^{1}(\Omega)^{d}}$ for all $w \in \boldsymbol{V}$. Moreover, for all $n \in \mathbb{N}, y^{n+1}$ satisfies

$$
\left\|y^{n+1}\right\|_{V}^{2} \leq \frac{c_{0}}{\nu}\left\|f^{n}\right\|_{H^{-1}(\Omega)^{d}}^{2}+\alpha\left\|y^{n}\right\|_{2}^{2}
$$

where $c_{0}>0$, only connected to the Poincaré constant, depends on $\Omega$. Moreover, for all $n \in \mathbb{N}^{\star}$ :

$$
\left\|y^{n}\right\|_{2}^{2}+\frac{\nu}{\alpha} \sum_{k=1}^{n}\left\|\nabla y^{k}\right\|_{2}^{2} \leq \frac{1}{\nu}\left(\frac{c_{0}}{\alpha} \sum_{k=0}^{n-1}\left\|f^{k}\right\|_{H^{-1}(\Omega)^{d}}^{2}+\nu\left\|u_{0}\right\|_{2}^{2}\right) .
$$

Proof. The existence of $y^{n+1}$ is given in Proposition 2.3 (3.7) is obtained by summing (3.6).

Remark 3.2. Arguing as in Lemma 2.4 if there exists a solution $y^{n+1}$ in $\boldsymbol{V}$ of (3.3) satisfying $\tau_{d}\left(y^{n+1}\right)<1$, then such solution is unique. In view of Proposition 3.1. this holds true if the quantity $\mathcal{M}(f, \alpha, \nu)$ defined as follows

$$
\mathcal{M}(f, \alpha, \nu)= \begin{cases}\frac{1}{\nu^{2}}\left(\frac{c_{0}}{\alpha} \sum_{k=0}^{n-1}\left\|f^{k}\right\|_{H^{-1}(\Omega)^{2}}^{2}+\nu\left\|u_{0}\right\|_{2}^{2}\right), & \text { if } \quad d=2, \\ \frac{\alpha^{1 / 2}}{\nu^{5 / 2}}\left(\frac{c_{0}}{\alpha} \sum_{k=0}^{n-1}\left\|f^{k}\right\|_{H^{-1}(\Omega)^{3}}^{2}+\nu\left\|u_{0}\right\|_{2}^{2}\right), & \text { if } \quad d=3\end{cases}
$$

is small enough.

We now distinguish the case $d=2$ from the case $d=3$ and consider either weak or regular solutions. 
3.1. Two dimensional case. We have the following convergence for weak solutions of (3.5).

Theorem 3.3. Suppose $F \in L^{2}\left(0, T ; H^{-1}(\Omega)^{2}\right), u_{0} \in \boldsymbol{V}$ and let $c\left(u_{0}, F\right)$ be defined as follows : $c\left(u_{0}, F\right):=\max \left(\frac{1}{\alpha}\left\|u_{0}\right\|_{\boldsymbol{V}}^{2}\left(\nu+\left\|u_{0}\right\|_{2}\right)^{2}+c_{0}\|F\|_{L^{2}\left(0, T ; H^{-1}(\Omega)^{2}\right)}^{2}, 2 c_{0}\|F\|_{L^{2}\left(0, T ; H^{-1}(\Omega)^{2}\right)}^{2}+\nu\left\|u_{0}\right\|_{2}^{2}\right)$.

Let $\alpha$ be large enough and $f^{n}$ be given 1.4 by all $n \in\{0, \cdots, N-1\}$ and let $\left\{y^{n}\right\}_{n \in \mathbb{N}}$ in $\boldsymbol{V}$ be the solution of 3.5). If there exists a constant $c>0$ such that

$$
c\left(u_{0}, F\right) \leq c \nu^{4}
$$

then, for any $n \geq 0$, the minimizing sequence $\left\{y_{k}^{n+1}\right\}_{k \in \mathbb{N}}$ defined by (3.4) strongly converges to the unique of solution of (3.5).

Proof. According to Proposition 2.13, we have to prove the existence of a constant $c_{1} \in(0,1)$ such that, for all $n \in\{0, \cdots, N-1\}$ and all $k \in \mathbb{N}, \tau_{2}\left(y_{k}^{n}\right) \leq c_{1}$.

For $n=0$, as in the previous section, it suffices to take $\alpha$ large enough to ensure the conditions 2.39 with $g=y_{0}^{0}=u_{0}$ leading to the property $\tau_{2}\left(y_{k}^{0}\right)<c_{1}$ for all $k \in \mathbb{N}$ and therefore $\tau_{2}\left(y^{1}\right)<c_{1}$.

For the next minimizing sequences, let us recall (see Lemma 2.14) that for all $n \in\{0, \cdots, N-$ 1) and all $k \in \mathbb{N}$

$$
\left\|y_{k}^{n+1}\right\|_{\boldsymbol{V}} \leq\left\|y^{n}\right\|_{\boldsymbol{V}}+\frac{m \sqrt{2}}{\nu\left(1-c_{1}\right)} \frac{\sqrt{E_{n}\left(y^{n}\right)}}{1-p_{n, 0}\left(\widetilde{\lambda}_{n, 0}\right)}
$$

where $p_{n, 0}\left(\widetilde{\lambda}_{n, 0}\right)$ is defined as in the proof of Proposition 2.7

First, since for all $n \in\{0, \cdots, N-1\},\left\|f^{n}\right\|_{H^{-1}(\Omega)^{2}}^{2} \leq \alpha\|F\|_{L^{2}\left(0, T ; H^{-1}(\Omega)^{2}\right)}^{2}$, we can write

$$
\begin{aligned}
E_{0}\left(y^{0}\right)=E_{0}\left(u_{0}\right) & \leq \frac{1}{2 \nu}\left(\left\|u_{0}\right\|_{\boldsymbol{V}}\left(\nu+\left\|u_{0}\right\|_{2}\right)+\sqrt{c_{0}}\left\|f^{0}\right\|_{H^{-1}(\Omega)^{2}}\right)^{2} \\
& \leq \frac{1}{\nu}\left(\left\|u_{0}\right\|_{\boldsymbol{V}}^{2}\left(\nu+\left\|u_{0}\right\|_{2}\right)^{2}+c_{0}\left\|u_{0}\right\|_{H^{-1}(\Omega)^{2}}^{2}\right) \\
& \leq \frac{\alpha}{\nu}\left(\frac{1}{\alpha}\left\|u_{0}\right\|_{\boldsymbol{V}}^{2}\left(\nu+\left\|u_{0}\right\|_{2}\right)^{2}+c_{0}\|F\|_{L^{2}\left(0, T ; H^{-1}(\Omega)^{2}\right)}^{2}\right) .
\end{aligned}
$$

Since $y^{n}$ is solution of 3.5 , it follows from 3.3 , that for all $n \in\{1, \cdots, N-1\}$ :

$$
\begin{aligned}
E_{n}\left(y^{n}\right) & \leq \frac{c_{0}}{2 \nu}\left\|f^{n}-f^{n-1}\right\|_{H^{-1}(\Omega)^{2}}^{2}+\frac{\alpha}{2}\left\|y^{n}-y^{n-1}\right\|_{2}^{2} \\
& \leq \frac{\alpha}{\nu}\left(2 c_{0}\|F\|_{L^{2}\left(0, T ; H^{-1}(\Omega)^{2}\right)}^{2}+\nu\left\|u_{0}\right\|_{2}^{2}\right) .
\end{aligned}
$$

Therefore, for all $n \in\{0, \cdots, N-1\}, E_{n}\left(y^{n}\right) \leq \frac{\alpha}{\nu} c\left(u_{0}, F\right)$.

Let $c_{1} \in(0,1)$ and suppose that $c\left(u_{0}, F\right)<\left(1-c_{1}\right)^{4} \nu^{3}$. Then, for any $K \in(0,1)$, there exists $\alpha_{0}>0$ such that, for all $\alpha \geq \alpha_{0}, c_{\alpha, \nu} \sqrt{E_{n}\left(y^{n}\right)} \leq K<1$. We therefore have (see Lemma 2.14), for all $\alpha \geq \alpha_{0}$, all $n \in\{0, \cdots, N-1\}$ and all $k \in \mathbb{N}$ :

$$
\begin{aligned}
\left\|y_{k}^{n+1}\right\|_{\boldsymbol{V}} & \leq\left\|y^{n}\right\|_{\boldsymbol{V}}+\frac{m \sqrt{2}}{\nu\left(1-c_{1}\right)} \frac{\sqrt{E_{n}\left(y^{n}\right)}}{1-c_{\alpha, \nu} \sqrt{E_{n}\left(y^{n}\right)}} \\
& \leq\left\|y^{n}\right\|_{\boldsymbol{V}}+\frac{m \sqrt{2}}{\nu\left(1-c_{1}\right)} \frac{\sqrt{E_{n}\left(y^{n}\right)}}{1-K} \\
& \leq\left\|y^{n}\right\|_{\boldsymbol{V}}+\frac{m \sqrt{2 \alpha}}{\nu^{3 / 2}\left(1-c_{1}\right)(1-K)} \sqrt{c\left(u_{0}, F\right)} .
\end{aligned}
$$

From 3.7 we then obtain, for all $n \in\{0, \cdots, N-1\}$,

$$
\left\|y^{n}\right\|_{\boldsymbol{V}} \leq \frac{\sqrt{\alpha}}{\nu} \sqrt{\frac{c_{0}}{\alpha} \sum_{k=0}^{n-1}\left\|f^{k}\right\|_{H^{-1}(\Omega)^{2}}^{2}+\nu\left\|u_{0}\right\|_{2}^{2}}
$$


and since $\frac{c_{0}}{\alpha} \sum_{k=0}^{n-1}\left\|f^{k}\right\|_{H^{-1}(\Omega)^{2}}^{2} \leq c_{0}\|F\|_{L^{2}\left(0, T ; H^{-1}(\Omega)^{2}\right)}^{2}$, we deduce that if

$$
c_{0}\|F\|_{L^{2}\left(0, T ; H^{-1}(\Omega)^{2}\right)}^{2}+\nu\left\|u_{0}\right\|_{2}^{2} \leq \frac{c_{1}^{2}}{2} \nu^{3}
$$

then $\left\|y^{n}\right\|_{V} \leq \frac{c_{1}}{2} \sqrt{2 \alpha \nu}$. Moreover, assuming $c\left(u_{0}, F\right) \leq \frac{c_{1}^{2}\left(1-c_{1}\right)^{2}(1-K)^{2}}{4 m} \nu^{4}$, we deduce from 3.10, for all $n \in\{0, \cdots, N-1\}$ and for all $k \in \mathbb{N}$ :

$$
\left\|y_{k}^{n}\right\|_{\boldsymbol{V}} \leq \frac{c_{1}}{2} \sqrt{2 \alpha \nu}+\frac{c_{1}}{2} \sqrt{2 \alpha \nu}=c_{1} \sqrt{2 \alpha \nu}
$$

that is $\tau_{2}\left(y_{k}^{n}\right) \leq c_{1}$. The result follows from Proposition 2.13

We emphasize that, for each $n \in \mathbb{N}$, the limit $y^{n+1}$ of the sequence $\left\{y_{k}^{n+1}\right\}_{k \in \mathbb{N}}$ satisfies $\tau_{2}\left(y^{n+1}\right)<1$ and is therefore the unique solution of 3.5 . Moreover, for $\alpha$ large enough, the condition (3.9) reads as the following smallness property on the data $u_{0}$ and $F: c_{0}\|F\|_{L^{2}\left(0, T ; H^{-1}(\Omega)^{2}\right)^{2}}^{2}+$ $\nu\left\|u_{0}\right\|_{2}^{2} \leq c \nu^{4}$. In contrast with the static case of Section (2) where the unique condition (2.39) on the data $g$ is fulfilled as soon as $\alpha$ is large, the iterated case requires a condition on the data $u_{0}$ and $F$, whatever be the amplitude of $\alpha$. Again, this smallness property is introduced in order to guarantees the condition $\tau_{2}\left(y^{n}\right)<1$ for all $n$. In view of (3.7), this condition implies notably that $\left\|y^{n}\right\|_{2} \leq c \nu^{3 / 2}$ for all $n>0$.

For regular solutions of (3.5) which we now consider, we may slightly improve the results, notably based on the control of two consecutive elements of the corresponding sequence $\left\{y^{n}\right\}_{n \in \mathbb{N}}$ for the $L^{2}(\Omega)$ norm. We first start with the following result of regularity.

Proposition 3.4. Assume that $\Omega$ is $C^{2}$, that $\left(f^{n}\right)_{n}$ is a sequence in $L^{2}(\Omega)^{2}$ and that $u_{0} \in \boldsymbol{V}$. Then, for all $n \in \mathbb{N}$, any solution $y^{n+1} \in \boldsymbol{V}$ of (3.5) belongs to $H^{2}(\Omega)^{2}$.

If moreover, there exists $C>0$ such that

$$
\frac{c_{0}}{\alpha} \sum_{k=0}^{n}\left\|f^{k}\right\|_{H^{-1}(\Omega)^{2}}^{2}+\nu\left\|y^{0}\right\|_{2}^{2}<C \nu^{3},
$$

then $y^{n+1}$ satisfies

$$
\int_{\Omega}\left|\nabla y^{n+1}\right|^{2}+\frac{\nu}{2 \alpha} \sum_{k=1}^{n+1} \int_{\Omega}\left|P \Delta y^{k}\right|^{2} \leq \frac{1}{\nu}\left(\frac{1}{\alpha} \sum_{k=0}^{n}\left\|f^{k}\right\|_{2}^{2}+\nu\left\|\nabla u_{0}\right\|_{2}^{2}\right)
$$

where $P$ is the operator of projection from $L^{2}(\Omega)^{d}$ into $\boldsymbol{H}$.

Proof. From Proposition 2.3 we know that for all $n \in \mathbb{N}^{*}, y^{n} \in H^{2}(\Omega)^{2} \cap \boldsymbol{V}$. Taking $w=P \Delta y^{n+1}$ in $(3.5)$ leads to :

$\alpha \int_{\Omega}\left|\nabla y^{n+1}\right|^{2}+\nu \int_{\Omega}\left|P \Delta y^{n+1}\right|^{2}=-\int_{\Omega} f^{n} P \Delta y^{n+1}+\int_{\Omega} y^{n+1} \cdot \nabla y^{n+1} \cdot P \Delta y^{n+1}+\alpha \int_{\Omega} \nabla y^{n} \cdot \nabla y^{n+1}$.

Recall that

$$
\int_{\Omega} f^{n} P \Delta y^{n+1} \leq \frac{1}{2 \nu}\left\|f^{n}\right\|_{2}^{2}+\frac{\nu}{2}\left\|P \Delta y^{n+1}\right\|_{2}^{2}, \quad \alpha \int_{\Omega} \nabla y^{n} \cdot \nabla y^{n+1} \leq \frac{\alpha}{2}\left\|y^{n}\right\|_{\boldsymbol{V}}^{2}+\frac{\alpha}{2}\left\|y^{n+1}\right\|_{\boldsymbol{V}}^{2} .
$$

We also have

$$
\left|\int_{\Omega} y^{n+1} \cdot \nabla y^{n+1} \cdot P \Delta y^{n+1}\right| \leq\left\|y^{n+1}\right\|_{\infty}\left\|\nabla y^{n+1}\right\|_{2}\left\|P \Delta y^{n+1}\right\|_{2} .
$$

We now use (see [18, chapter 5]) that there exist three constants $c_{1}, c_{2}$ and $c_{3}$ such that

$$
\left\|\Delta y^{n+1}\right\|_{2} \leq c_{1}\left\|P \Delta y^{n+1}\right\|_{2}, \quad\left\|y^{n+1}\right\|_{\infty} \leq c_{2}\left\|y^{n+1}\right\|_{2}^{\frac{1}{2}}\left\|\Delta y^{n+1}\right\|_{2}^{\frac{1}{2}}
$$

and

$$
\left\|\nabla y^{n+1}\right\|_{2} \leq c_{3}\left\|y^{n+1}\right\|_{2}^{\frac{1}{2}}\left\|\Delta y^{n+1}\right\|_{2}^{\frac{1}{2}}
$$


This implies that

$$
\left|\int_{\Omega} y^{n+1} \cdot \nabla y^{n+1} \cdot P \Delta y^{n+1}\right| \leq c\left\|y^{n+1}\right\|_{2}\left\|P \Delta y^{n+1}\right\|_{2}^{2}
$$

with $c=c_{1} c_{2} c_{3}$. Recalling 3.13 , it follows that

$$
\frac{\alpha}{2} \int_{\Omega}\left|\nabla y^{n+1}\right|^{2}+\left(\frac{\nu}{2}-c\left\|y^{n+1}\right\|_{2}\right) \int_{\Omega}\left|P \Delta y^{n+1}\right|^{2} \leq \frac{1}{2 \nu}\left\|f^{n}\right\|_{2}^{2}+\frac{\alpha}{2} \int_{\Omega}\left|\nabla y^{n}\right|^{2} .
$$

But, from estimate (3.7), the assumption 3.11 implies that $\left\|y^{n+1}\right\|_{2} \leq \frac{\nu}{4 c}$ and

$$
\int_{\Omega}\left|\nabla y^{n+1}\right|^{2}+\frac{\nu}{2 \alpha} \int_{\Omega}\left|P \Delta y^{n+1}\right|^{2} \leq \frac{1}{\nu \alpha}\left\|f^{n}\right\|_{2}^{2}+\int_{\Omega}\left|\nabla y^{n}\right|^{2} .
$$

Summing then implies 3.12 for all $n \in \mathbb{N}$.

Remark 3.5. Under the hypothesis of Proposition 3.4. suppose that

$$
B_{\alpha, \nu}:=\left(\alpha \nu^{5}\right)^{-1}\left(c_{0} \alpha^{-1} \sum_{k=0}^{n}\left\|f^{k}\right\|_{H^{-1}(\Omega)^{2}}^{2}+\nu\left\|y^{0}\right\|_{2}^{2}\right)\left(\alpha^{-1} \sum_{k=0}^{n-1}\left\|f^{k}\right\|_{2}^{2}+\nu\left\|\nabla y^{0}\right\|_{2}^{2}\right)
$$

is small (which is satisfied as soon as $\alpha$ is large enough). Then, the solution of (3.5) is unique.

Indeed, let $n \in \mathbb{N}$ and let $y_{1}^{n+1}, y_{2}^{n+1} \in \boldsymbol{V}$ be two solutions of (3.5). Then $Y:=y_{1}^{n+1}-y_{2}^{n+1}$ satisfies

$$
\alpha \int_{\Omega} Y \cdot w+\nu \int_{\Omega} \nabla Y \cdot \nabla w+\int_{\Omega} y_{2}^{n+1} \cdot \nabla Y \cdot w+\int_{\Omega} Y \cdot \nabla y_{1}^{n+1} \cdot w=0 \quad \forall w \in \boldsymbol{V}
$$

and in particular, for $w=Y\left(\right.$ since $\left.\int_{\Omega} y_{2}^{n+1} \cdot \nabla Y \cdot Y=0\right)$

$$
\begin{aligned}
\alpha \int_{\Omega}|Y|^{2}+\nu \int_{\Omega}|\nabla Y|^{2} & =-\int_{\Omega} Y \cdot \nabla y_{1}^{n+1} \cdot Y=\int_{\Omega} Y \cdot \nabla Y \cdot y_{1}^{n+1} \leq c\left\|y_{1}^{n+1}\right\|_{\infty}\|\nabla Y\|_{2}\|Y\|_{2} \\
& \leq c\left\|y_{1}^{n+1}\right\|_{2}^{1 / 2}\left\|P \Delta y_{1}^{n+1}\right\|_{2}^{1 / 2}\|\nabla Y\|_{2}\|Y\|_{2} \\
& \leq \alpha\|Y\|_{2}^{2}+\frac{c}{\alpha}\left\|y_{1}^{n+1}\right\|_{2}\left\|P \Delta y_{1}^{n+1}\right\|_{2}\|\nabla Y\|_{2}^{2}
\end{aligned}
$$

leading to

$$
\left(\nu-\frac{c}{\alpha}\left\|y_{1}^{n+1}\right\|_{2}\left\|P \Delta y_{1}^{n+1}\right\|_{2}\right)\|\nabla Y\|_{2}^{2} \leq 0
$$

If

$$
\left\|y_{1}^{n+1}\right\|_{2}\left\|P \Delta y_{1}^{n+1}\right\|_{2}<\frac{\nu \alpha}{c}
$$

then $Y=0$ and the solution is unique. But, from (3.7) and (3.12),

$$
\left\|y_{1}^{n+1}\right\|_{2}^{2}\left\|P \Delta y_{1}^{n+1}\right\|_{2}^{2} \leq \frac{4 \alpha}{\nu^{3}}\left(\frac{c_{0}}{\alpha} \sum_{k=0}^{n}\left\|f^{k}\right\|_{H^{-1}(\Omega)^{2}}^{2}+\nu\left\|y^{0}\right\|_{2}^{2}\right)\left(\frac{1}{\alpha} \sum_{k=0}^{n}\left\|f^{k}\right\|_{2}^{2}+\nu\left\|\nabla y^{0}\right\|_{2}^{2}\right) .
$$

Therefore, there exists a constant $C$ such that if $B_{\alpha, \nu}<C$, then (3.14) holds true.

Proposition 3.4 then allows to obtain the following estimate of $\left\|y^{n+1}-y^{n}\right\|_{2}$ in term of the parameter $\alpha$.

Theorem 3.6. We assume that $\Omega$ is $C^{2}$, that $\left(f^{n}\right)_{n}$ is a sequence in $L^{2}(\Omega)^{2}$ satisfies $\alpha^{-1} \sum_{k=0}^{+\infty}\left\|f^{k}\right\|_{2}^{2}<$ $+\infty$, that $u_{0} \in \boldsymbol{V}$ and that for all $n \in \mathbb{N}$, $y^{n+1} \in H^{2}(\Omega)^{2} \cap \boldsymbol{V}$ is a solution of (3.5) satisfying $\left\|y^{n+1}\right\|_{2} \leq \frac{\nu}{4 c}$. Then, there exists $C_{1}>0$ such that the sequence $\left(y^{n}\right)_{n}$ satisfies

$$
\left\|y^{n+1}-y^{n}\right\|_{2}^{2} \leq \frac{C_{1}}{\alpha \nu^{3 / 2}} .
$$


Proof. For all $n \in \mathbb{N}, w=y^{n+1}-y^{n}$ in 3.5 gives :

$\alpha\left\|y^{n+1}-y^{n}\right\|_{2}^{2}+\nu\left\|\nabla y^{n+1}\right\|_{2}^{2} \leq\left|\int_{\Omega} y^{n+1} \cdot \nabla y^{n+1} \cdot\left(y^{n+1}-y^{n}\right)\right|+\left|\int_{\Omega} f^{n} \cdot\left(y^{n+1}-y^{n}\right)\right|+\nu\left|\int_{\Omega} \nabla y^{n} \cdot \nabla y^{n+1}\right|$.

Moreover,

$\left.\left|\int_{\Omega} y^{n+1} \cdot \nabla y^{n+1} \cdot\left(y^{n+1}-y^{n}\right)\right| \leq c\left\|\nabla y^{n+1}\right\|_{2}^{2}\left\|\nabla\left(y^{n+1}-y^{n}\right)\right\|_{2} \leq c\left\|\nabla y^{n+1}\right\|_{2}^{2}\left(\left\|\nabla y^{n+1}\right\|_{2}+\| \nabla y^{n}\right) \|_{2}\right)$.

Therefore,

$$
\alpha\left\|y^{n+1}-y^{n}\right\|_{2}^{2}+\nu\left\|\nabla y^{n+1}\right\|_{2}^{2} \leq c\left\|\nabla y^{n+1}\right\|_{2}^{2}\left(\left\|\nabla y^{n+1}\right\|_{2}+\left\|\nabla y^{n}\right\|_{2}\right)+\frac{1}{\alpha}\left\|f^{n}\right\|_{2}^{2}+\nu\left\|\nabla y^{n}\right\|_{2}^{2} .
$$

But, 3.12 implies that for all $n \in \mathbb{N}$

$$
\int_{\Omega}\left|\nabla y^{n+1}\right|^{2} \leq \frac{1}{\nu}\left(\frac{1}{\alpha} \sum_{k=0}^{+\infty}\left\|f^{k}\right\|_{2}^{2}+\nu\left\|\nabla y^{0}\right\|_{2}^{2}\right):=\frac{C}{\nu}
$$

and thus, since $\nu<1$

$$
\alpha\left\|y^{n+1}-y^{n}\right\|_{2}^{2}+\nu\left\|\nabla y^{n+1}\right\|_{2}^{2} \leq \frac{2 c C^{3 / 2}}{\nu^{3 / 2}}+2 C \leq \frac{C_{1}}{\nu^{3 / 2}}
$$

leading to $\left\|y^{n+1}-y^{n}\right\|_{2}^{2}=O\left(\frac{1}{\alpha \nu^{3 / 2}}\right)$ as announced.

This result asserts that two consecutive elements of the sequence $\left\{y^{n}\right\}_{n \geq 0}$ defined by recurrence from the scheme 1.2 are close each other as soon as $\delta t$, the time step discretization, is small enough. In particular, this justifies the choice of the initial term $y_{0}^{n+1}=y^{n}$ of the minimizing sequence in order to approximate $y^{n+1}$.

We end this section devoted to the case $d=2$ with the following result, analogue of Theorem (3.3), for regular data.

Theorem 3.7. Suppose $F \in L^{2}\left(0, T ; L^{2}(\Omega)^{2}\right), u_{0} \in \boldsymbol{V}$, for all $n \in\{0, \cdots, N-1\}$, $\alpha$ and $f^{n}$ are given by (1.4) and $y^{n+1} \in \boldsymbol{V}$ solution of (3.5). If $C\left(u_{0}, F\right):=\|F\|_{L^{2}\left(0, T ; L^{2}(\Omega)^{2}\right)}^{2}+\nu\left\|u_{0}\right\|_{\boldsymbol{V}}^{2} \leq C \nu^{2}$ for some $C$ and $\alpha$ is large enough, then, for any $n \geq 0$, the minimizing sequence $\left\{y_{k}^{n+1}\right\}_{k \in \mathbb{N}}$ defined by (3.4) strongly converges to the unique of solution of (3.5).

Proof. As for Theorem 3.3, it suffices to prove that there exists $c_{1} \in(0,1)$ such that, for all $n \in\{0, \cdots, N-1\}$ and all $k \in \mathbb{N}, \tau_{2}\left(y_{k}^{n}\right) \leq c_{1}$. Let us recall that for all $n \in\{0, \cdots, N-1\}$ and all $k \in \mathbb{N}$

$$
\left\|y_{k+1}^{n+1}\right\|_{\boldsymbol{V}} \leq\left\|y^{n}\right\|_{\boldsymbol{V}}+\frac{m \sqrt{2}}{\nu\left(1-c_{1}\right)} \frac{\sqrt{E_{n}\left(y^{n}\right)}}{1-p_{n, 0}\left(\widetilde{\lambda}_{n, 0}\right)}
$$

where $p_{n, 0}\left(\widetilde{\lambda}_{n, 0}\right)$ is defined as in the proof of Proposition 2.7. From 3.3, since for all $n \in$ $\{0, \cdots, N-1\},\left\|f^{n}\right\|_{2}^{2} \leq \alpha\|F\|_{L^{2}\left(0, T ; L^{2}(\Omega)^{2}\right)}^{2}:$

$$
\begin{aligned}
E_{0}\left(y^{0}\right)=E_{0}\left(u_{0}\right) & \leq \frac{1}{2 \nu}\left(\left\|u_{0}\right\|_{\boldsymbol{V}}\left(\nu+\left\|u_{0}\right\|_{2}\right)+\sqrt{\frac{\nu}{\alpha}}\left\|f^{1}\right\|_{2}\right)^{2} \\
& \leq \frac{1}{\nu}\left\|u_{0}\right\|_{\boldsymbol{V}}^{2}\left(\nu+\left\|u_{0}\right\|_{2}\right)^{2}+\|F\|_{L^{2}\left(0, T ; L^{2}(\Omega)^{2}\right)}^{2}
\end{aligned}
$$

and, since $y^{n}$ is solution of 3.5 , then for all $n \in\{1, \cdots, N-1\}$ :

$$
\begin{aligned}
E_{n}\left(y^{n}\right) & \leq \frac{1}{\alpha}\left\|f^{n}-f^{n-1}\right\|_{2}^{2}+\alpha\left\|y^{n}-y^{n-1}\right\|_{2}^{2} \\
& \leq 2\|F\|_{L^{2}\left(0, T ; L^{2}(\Omega)^{2}\right)}^{2}+\alpha\left\|y^{n}-y^{n-1}\right\|_{2}^{2} .
\end{aligned}
$$

From the proof of Theorem 3.6, we deduce that for all $n \in\{0, \cdots, N-1\}$ :

$$
\alpha\left\|y^{n+1}-y^{n}\right\|_{2}^{2} \leq \frac{2 c C\left(u_{0}, F\right)^{3 / 2}}{\nu^{3 / 2}}+2 C\left(u_{0}, F\right)
$$


and thus, for all $n \in\{1, \cdots, N-1\}$

$$
E_{n}\left(y^{n}\right) \leq \frac{2 c C\left(u_{0}, F\right)^{3 / 2}}{\nu^{3 / 2}}+4 C\left(u_{0}, F\right) .
$$

Moreover, from 3.12 , for all $n \in\{0, \cdots, N-1\}$ :

$$
\left\|y^{n}\right\|_{\boldsymbol{V}}^{2} \leq \frac{1}{\nu}\left(\frac{1}{\alpha} \sum_{k=0}^{n}\left\|f^{k}\right\|_{2}^{2}+\nu\left\|u_{0}\right\|_{\boldsymbol{V}}^{2}\right) \leq \frac{1}{\nu}\left(\|F\|_{L^{2}\left(0, T ; L^{2}(\Omega)^{2}\right)}^{2}+\nu\left\|u_{0}\right\|_{\boldsymbol{V}}^{2}\right)=\frac{1}{\nu} C\left(u_{0}, F\right) .
$$

Eventually, let $c_{1} \in(0,1)$. Then there exists $\alpha_{0}>0$ such that, for all $\alpha \geq \alpha_{0} c_{\alpha, \nu} \sqrt{E_{n}\left(y^{n}\right)} \leq$ $K<1$. We therefore have (see Theorem 3.3), for all $\alpha \geq \alpha_{0}$, all $n \in\{0, \cdots, N-1\}$ and all $k \in \mathbb{N}$ :

$$
\left\|y_{k+1}^{n+1}\right\|_{\boldsymbol{V}} \leq\left\|y^{n}\right\|_{\boldsymbol{V}}+\frac{m \sqrt{2}}{\nu\left(1-c_{1}\right)} \frac{\sqrt{E_{n}\left(y^{n}\right)}}{1-K}
$$

which gives a bound of $\left\|y_{k+1}^{n+1}\right\|_{\boldsymbol{V}}$ independent of $\alpha \geq \alpha_{0}$.

Taking $\alpha_{1} \geq \alpha_{0}$ large enough, we deduce that, for all $\alpha \geq \alpha_{1}$, all $n \in\{0, \cdots, N-1\}$ and all $k \in \mathbb{N},\left\|y_{k}^{n}\right\|_{\boldsymbol{V}} \leq c_{1} \sqrt{2 \alpha \nu}$, that is $\tau_{2}\left(y_{k}^{n}\right) \leq c_{1}$. The announced convergence follows from Proposition 2.13 .

3.2. Three dimensional case. We now consider regular solutions for the case $d=3$. The following intermediate regularity result holds true.

Proposition 3.8. Assume that $\Omega$ is $C^{2}$, that $\left(f^{n}\right)_{n}$ is a sequence in $L^{2}(\Omega)^{3}$ and that $u_{0} \in \boldsymbol{V}$. Then any solution $y^{n+1} \in \boldsymbol{V}$ of (3.5) belongs to $H^{2}(\Omega)^{3}$.

If moreover, there exists $C>0$ such that

$$
\frac{1}{\alpha} \sum_{k=0}^{n}\left\|f^{k}\right\|_{2}^{2}+\nu \int_{\Omega}\left|\nabla u_{0}\right|^{2} \leq C \nu^{3}
$$

then the inequality (3.12) holds true.

Proof. From Proposition 2.3 we know that for all $n \in \mathbb{N}^{*}, y^{n} \in H^{2}(\Omega)^{3} \cap \boldsymbol{V}$. Let now $P$ be the operator of projection from $L^{2}(\Omega)^{3}$ into $\boldsymbol{H}$. Taking $w=P \Delta y^{n+1}$ in 3.5 leads to :

$\alpha \int_{\Omega}\left|\nabla y^{n+1}\right|^{2}+\nu \int_{\Omega}\left|P \Delta y^{n+1}\right|^{2}=-\int_{\Omega} f^{n} \cdot P \Delta y^{n+1}+\int_{\Omega} y^{n+1} \cdot \nabla y^{n+1} \cdot P \Delta y^{n+1}+\alpha \int_{\Omega} \nabla y^{n} \cdot \nabla y^{n+1}$.

In view of the inequality

$$
\left|\int_{\Omega} y^{n+1} \cdot \nabla y^{n+1} \cdot P \Delta y^{n+1}\right| \leq\left\|y^{n+1}\right\|_{3}\left\|\nabla y^{n+1}\right\|_{6}\left\|P \Delta y^{n+1}\right\|_{2},
$$

we use again that there exist constants $c_{1}, c_{2}>0$ such that

$$
\left\|\Delta y^{n+1}\right\|_{2} \leq c_{1}\left\|P \Delta y^{n+1}\right\|_{2}, \quad\left\|\nabla y^{n+1}\right\|_{6} \leq c_{2}\left\|\Delta y^{n+1}\right\|_{2} \leq c_{1} c_{2}\left\|P \Delta y^{n+1}\right\|_{2}
$$

so that, for $c=c_{1} c_{2}$, we obtain

$$
\left|\int_{\Omega} y^{n+1} \cdot \nabla y^{n+1} \cdot P \Delta y^{n+1}\right| \leq c\left\|y^{n+1}\right\|_{3}\left\|P \Delta y^{n+1}\right\|_{2}^{2} .
$$

It results from (3.17) that

$$
\frac{\alpha}{2} \int_{\Omega}\left|\nabla y^{n+1}\right|^{2}+\left(\frac{\nu}{2}-c\left\|y^{n+1}\right\|_{3}\right) \int_{\Omega}\left|P \Delta y^{n+1}\right|^{2} \leq \frac{1}{2 \nu}\left\|f^{n}\right\|_{2}^{2}+\frac{\alpha}{2} \int_{\Omega}\left|\nabla y^{n}\right|^{2} .
$$

Assume that, for all $n \in \mathbb{N}^{*}$, we have constructed by recurrence an element $y^{n}$ solution of 3.5 such that

$$
\frac{\nu}{4}-c\left\|y^{n}\right\|_{3}>0
$$


Then, for all $n \in \mathbb{N}$

$$
\int_{\Omega}\left|\nabla y^{n+1}\right|^{2}+\frac{\nu}{2 \alpha} \int_{\Omega}\left|P \Delta y^{n+1}\right|^{2} \leq \frac{1}{\nu \alpha}\left\|f^{n}\right\|_{2}^{2}+\int_{\Omega}\left|\nabla y^{n}\right|^{2}
$$

and recursively, for all $n \in \mathbb{N}^{*}$ :

$$
\int_{\Omega}\left|\nabla y^{n+1}\right|^{2}+\frac{\nu}{2 \alpha} \sum_{k=1}^{n+1} \int_{\Omega}\left|P \Delta y^{k}\right|^{2} \leq \frac{1}{\nu}\left(\frac{1}{\alpha} \sum_{k=0}^{n}\left\|f^{k}\right\|_{2}^{2}+\nu \int_{\Omega}\left|\nabla u_{0}\right|^{2}\right)
$$

It remains to construct a sequence $\left(y^{n}\right)_{n \in \mathbb{N}^{*}}$ solution of 3.5 and satisfying for all $n \in \mathbb{N}^{*}$ the property (3.19). Let $n \in \mathbb{N}^{*}$ fixed. Assume now, that we have constructed, for $k \in\{1, \cdots, n\}$ a solution $y^{k}$ satisfying 3.5 and $\frac{\nu}{4}-c\left\|y^{k}\right\|_{3}>0$ for $c=c_{1} c_{2}$ introduced above. Let $y_{1} \in \boldsymbol{V}$ and let $y_{2} \in H^{2}(\Omega)^{3} \cap \boldsymbol{V}$ be the unique solution of

$$
\alpha \int_{\Omega}\left(y_{2}-y^{n}\right) \cdot w+\nu \int_{\Omega} \nabla y_{2} \cdot \nabla w+\int_{\Omega} y_{1} \cdot \nabla y_{2} \cdot w=\left\langle f^{n}, w\right\rangle_{H^{-1}(\Omega)^{d} \times H_{0}^{1}(\Omega)^{d}} \quad \forall w \in \boldsymbol{V} .
$$

If $y_{1}$ satisfies $\frac{\nu}{4 c} \geq\left\|y_{1}\right\|_{3}$, then in view of 3.18 ,

$$
\frac{\alpha}{2} \int_{\Omega}\left|\nabla y_{2}\right|^{2}+\left(\frac{\nu}{2}-c\left\|y_{1}\right\|_{3}\right) \int_{\Omega}\left|P \Delta y_{2}\right|^{2} \leq \frac{1}{2 \nu}\left\|f^{n}\right\|_{2}^{2}+\frac{\alpha}{2} \int_{\Omega}\left|\nabla y^{n}\right|^{2}
$$

and consequently

$$
\frac{\alpha}{2} \int_{\Omega}\left|\nabla y_{2}\right|^{2}+\frac{\nu}{4} \int_{\Omega}\left|P \Delta y_{2}\right|^{2} \leq \frac{1}{2 \nu}\left\|f^{n}\right\|_{2}^{2}+\frac{\alpha}{2} \int_{\Omega}\left|\nabla y^{n}\right|^{2} .
$$

3.20 then implies

$$
\int_{\Omega}\left|\nabla y_{2}\right|^{2}+\frac{\nu}{2 \alpha} \int_{\Omega}\left|P \Delta y_{2}\right|^{2} \leq \frac{1}{\nu}\left(\frac{1}{\alpha} \sum_{k=0}^{n}\left\|f^{k}\right\|_{2}^{2}+\nu \int_{\Omega}\left|\nabla y^{0}\right|^{2}\right) .
$$

We now use that there exists a constant $c_{3}>0$ such that, for all $n \in \mathbb{N}\left\|y_{2}\right\|_{3} \leq c_{3}\left\|\nabla y_{2}\right\|_{2}$ to obtain

$$
\left\|y_{2}\right\|_{3}^{2} \leq \frac{c_{3}^{2}}{\nu}\left(\frac{1}{\alpha} \sum_{k=0}^{n}\left\|f^{k}\right\|_{2}^{2}+\nu \int_{\Omega}\left|\nabla u_{0}\right|^{2}\right) .
$$

Invoking assumption 3.16, we conclude that $\left\|y_{2}\right\|_{3} \leq \frac{\nu}{4 c}$.

Eventually, we introduce the application $\mathcal{T}: \mathcal{C} \rightarrow \mathcal{C}, y_{1} \mapsto y_{2}$ where $\mathcal{C}$ is the closed convex set of $\boldsymbol{V}$ defined by $\mathcal{C}:=\left\{y \in \boldsymbol{V}, \frac{\nu}{4 c} \geq\|y\|_{3}\right\}$. Let us check that $\mathcal{T}$ is continuous. Let $y_{1} \in \mathcal{C}$ et $z_{1} \in \mathcal{C}, y_{2}=\mathcal{T}\left(y_{1}\right)$ et $z_{2}=\mathcal{T}\left(z_{1}\right)$ so that

$\alpha \int_{\Omega}\left(z_{2}-y_{2}\right) \cdot w+\nu \int_{\Omega} \nabla\left(z_{2}-y_{2}\right) \cdot \nabla w+\int_{\Omega} y_{1} \cdot \nabla\left(y_{2}-z_{2}\right) \cdot w+\int_{\Omega}\left(y_{1}-z_{1}\right) \cdot \nabla z_{2} \cdot w=0 \quad \forall w \in \boldsymbol{V}$ and then for $w=z_{2}-y_{2}$

$$
\begin{aligned}
\alpha \int_{\Omega}\left|z_{2}-y_{2}\right|^{2}+\nu \int_{\Omega}\left|\nabla\left(z_{2}-y_{2}\right)\right|^{2} & \leq\left|\int_{\Omega}\left(y_{1}-z_{1}\right) \cdot \nabla z_{2} \cdot\left(z_{2}-y_{2}\right)\right| \\
& \leq c\left\|\nabla\left(y_{1}-z_{1}\right)\right\|_{2}\left\|\nabla z_{2}\right\|_{2}\left\|z_{2}-y_{2}\right\|_{3} \\
& \leq c\left\|\nabla\left(y_{1}-z_{1}\right)\right\|_{2}
\end{aligned}
$$

using (3.21); this implies the continuity of $\mathcal{T}$. On the other hand, since $\mathcal{T}(\mathcal{C})$ is a bounded set of $H^{2}(\Omega)^{3}, \mathcal{T}$ is relatively compact. The Schauder theorem allows to affirm that $\mathcal{T}$ has a fixe point $y \in \mathcal{C}$, that is, a solution $y^{n+1} \in \mathcal{C}$ of 3.5 .

Remark 3.9. Under the hypothesis of Proposition 3.8, suppose moreover that

$$
C_{\alpha, \nu}:=\nu^{-5 / 2} \alpha^{-1 / 2}\left(\alpha^{-1} \sum_{k=0}^{n-1}\left\|f^{k}\right\|_{2}^{2}+\nu\left\|\nabla y^{0}\right\|_{2}^{2}\right)
$$

is small enough, then, the solution of (3.5) is unique. 
Indeed, let $n \in \mathbb{N}$ and let $y_{1}^{n+1}, y_{2}^{n+1} \in \boldsymbol{V}$ be two solutions of (3.5). Let $Y:=y_{1}^{n+1}-y_{2}^{n+1}$. Then,

$$
\alpha \int_{\Omega} Y \cdot w+\nu \int_{\Omega} \nabla Y \cdot \nabla w+\int_{\Omega} y_{2}^{n+1} \cdot \nabla Y . w+\int_{\Omega} Y \cdot \nabla y_{1}^{n+1} \cdot w=0 \quad \forall w \in \boldsymbol{V}
$$

and in particular, for $w=Y\left(\right.$ since $\left.\int_{\Omega} y_{2}^{n+1} \cdot \nabla Y \cdot Y=0\right)$

$$
\begin{aligned}
\alpha \int_{\Omega}|Y|^{2}+\nu \int_{\Omega}|\nabla Y|^{2} & =-\int_{\Omega} Y \cdot \nabla y_{1}^{n+1} \cdot Y=\int_{\Omega} Y \cdot \nabla Y \cdot y_{1}^{n+1} \leq c\left\|y_{1}^{n+1}\right\|_{\infty}\|\nabla Y\|_{2}\|Y\|_{2} \\
& \leq c\left\|\nabla y_{1}^{n+1}\right\|_{2}^{1 / 2}\left\|P \Delta y_{1}^{n+1}\right\|_{2}^{1 / 2}\|\nabla Y\|_{2}\|Y\|_{2} \\
& \leq \alpha\|Y\|_{2}^{2}+\frac{c}{\alpha}\left\|\nabla y_{1}^{n+1}\right\|_{2}\left\|P \Delta y_{1}^{n+1}\right\|_{2}\|\nabla Y\|_{2}^{2}
\end{aligned}
$$

and therefore $\left(\nu-\frac{c}{\alpha}\left\|\nabla y_{1}^{n+1}\right\|_{2}\left\|P \Delta y_{1}^{n+1}\right\|_{2}\right)\|\nabla Y\|_{2}^{2} \leq 0$. Moreover, from 3.12),

$$
\left\|\nabla y_{1}^{n+1}\right\|_{2}\left\|P \Delta y_{1}^{n+1}\right\|_{2} \leq \frac{2 \alpha^{1 / 2}}{\nu^{3 / 2}}\left(\frac{1}{\alpha} \sum_{k=0}^{n}\left\|f^{k}\right\|_{2}^{2}+\nu\left\|\nabla y^{0}\right\|_{2}^{2}\right) .
$$

Therefore, there exists a constant $c>0$ such that if $C_{\alpha, \nu}<c$, then, arguing as in the 2d case, $\|\nabla Y\|_{2}^{2} \leq 0$ and $Y=0$.

As in the case $d=2$, Proposition 3.8 then allows, following the proof of Theorem 3.6, to obtain the following estimate of $\left\|y^{n+1}-y^{n}\right\|_{2}$ in term of the parameter $\alpha$.

Theorem 3.10. We assume that $\Omega$ is $C^{2}$, that $\left(f^{n}\right)_{n}$ is a sequence in $L^{2}(\Omega)^{3}$ satisfies $\alpha^{-1} \sum_{k=0}^{+\infty}\left\|f^{k}\right\|_{2}<$ $+\infty$, that $y^{0} \in \boldsymbol{V}$ and that for all $n \in \mathbb{N}, y^{n+1} \in H^{2}(\Omega)^{3} \cap \boldsymbol{V}$ is a solution of (3.5) satisfying 3.12). Then, the sequence $\left(y^{n}\right)_{n}$ satisfies 3.15.

Eventually, adapting the proof of Theorem 3.7, we get the following convergence result.

Theorem 3.11. Suppose $F \in L^{2}\left(0, T ; L^{2}(\Omega)^{3}\right), y^{0} \in \boldsymbol{V}$, for all $n \in\{0, \cdots, N-1\}$, $\alpha$ and $f^{n}$ are given by (1.4) and $y^{n+1} \in \boldsymbol{V}$ solution of (3.5). If $C\left(y^{0}, F\right):=\|F\|_{L^{2}\left(0, T ; L^{2}(\Omega)\right)}^{2}+\nu\left\|y^{0}\right\|_{\boldsymbol{V}}^{2} \leq C \nu^{3}$ for some $C>0$ and $\alpha$ is large enough, then for any $n \geq 0$, the minimizing sequence $\left\{y_{k}^{n+1}\right\}_{k \in \mathbb{N}}$ defined by (3.4) strongly converges to the unique of solution of (3.5).

Remark 3.12. We have considered regular solutions in the case $d=3$ in order to be able to prove the uniform property $\tau_{3}\left(y_{k}^{n}\right) \leq c_{1}<1$ for some $c_{1}$ independent of $k$ and $n$, i.e. $\left\|y_{k}^{n}\right\|_{\boldsymbol{V}} \leq$ $c_{1} M^{-1}\left(\alpha \nu^{3}\right)^{1 / 4}$. Actually, for regular solutions, Proposition 3.8 implies that $\left\|y_{k}^{n}\right\|_{\boldsymbol{V}} \leq C$ for some $C$ independent of $\alpha$, which is sufficient for $\alpha$ large enough. By considering weak solution, we can only prove that $\left\|y_{k}^{n}\right\|_{\boldsymbol{V}} \leq C \alpha^{1 / 2}$ for some $C>0$ (see (3.7)) which does not imply $\tau_{3}\left(y_{k}^{n}\right) \leq c_{1}<1$.

\section{Numerical illustrations}

We discuss in this section numerical experiments based on finite element approximations in space for two geometries of $\mathbb{R}^{2}$ : the celebrated channel with a backward facing step and the semicircular driven cavity introduced in [5]. In both cases, the velocity of the fluid is imposed on the boundary. We first start with the case $\alpha=0$ in (2.5) (discussed in [11]) allowing, first to get the solution of (1.1) as time becomes large and secondly, to enhance the gain of the optimization of the descent step parameter $\lambda_{k}$ in 2.22 . Then, for the semi driven cavity, we consider the cases $\alpha=0$ and $\alpha>0$ applied to the resolution of the backward Euler scheme (3.1). In a final part, we briefly compare the computational cost of this least-squares approach with standard explicit and semi-explicit scheme.

The numerical simulations are performed with the FreeFem ++ package developed at Sorbonne university (see [8]). Regular triangular meshes are used together with the $\mathbb{P}_{2} / \mathbb{P}_{1}$ Taylor-Hood finite element, satisfying the Ladyzenskaia-Babushka-Brezzi condition of stability. 
4.1. Steady case : Two dimensional channel with a backward facing step. We consider in the steady situation the celebrated test problem of a two-dimensional channel with a backward facing step, described for instance in Section 45 of [6] (see also 9]). We use exactly the geometry and boundary conditions from this reference. The geometry is depicted in Figure 1. Dirichlet conditions of the Poiseuille type are imposed on the entrant and sortant sides $\Gamma_{1}$ and $\Gamma_{2}$ of the channel: we impose $y=\left(4\left(H-x_{2}\right)\left(x_{2}-h\right) /(H-h)^{2}, 0\right)$ on $\Gamma_{1}$ and $y=\left(4(H-h) x_{2}\left(H-x_{2}\right) / H^{2}, 0\right)$ on $\Gamma_{2}$, with $h=1, H=3, l=3$ and $L=30$. On the remaining part $\partial \Omega \backslash\left(\Gamma_{1} \cup \Gamma_{2}\right)$, the fluid flow is imposed to zero. The external force $f$ is zero.

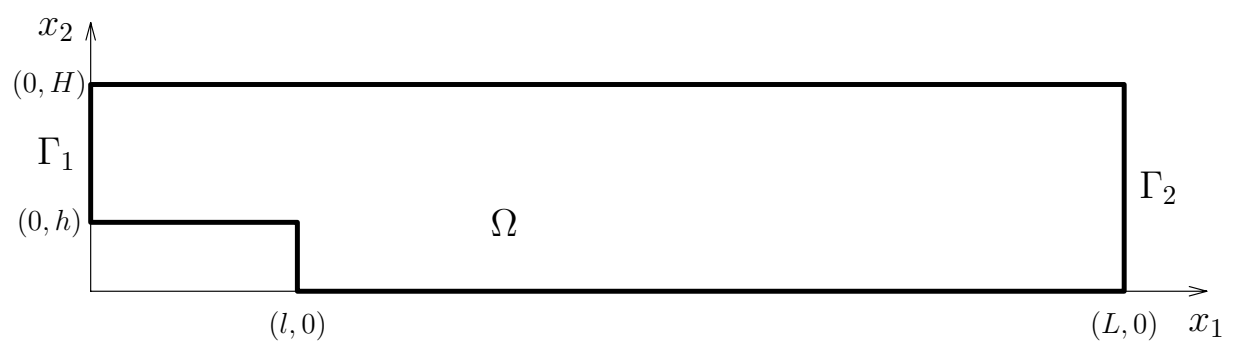

Figure 1. A two-dimensional channel with a step.

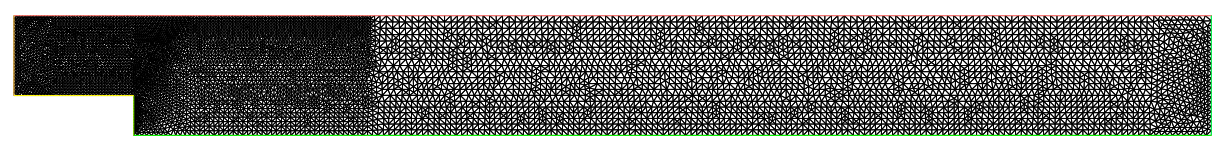

Figure 2. A triangular mesh of the channel - 14143 triangles and 7360 vertices.

We consider the extremal problem 2.7 to solve the steady Navier-Stokes equation (1.3) with here $\alpha=0$. We compare the descent algorithm (2.22) based on the descent direction $Y_{1}$ exhibited in the proof of Proposition 2.7 with the conjugate gradient (CG) algorithm used in [11. In both cases, the initial guess is defined as the solution of the corresponding Stokes problem. Moreover, the scalar extremal problem with respect to $\lambda_{k}$ in 2.22 is performed with the Newton-Rasphon method for real function.

We start with a relatively large value of $\nu=1 / 150$. Table 1 reports the evolution of the relative quantity $\left\|y_{k+1}-y_{k}\right\|_{\boldsymbol{V}} /\left\|y_{k}\right\|_{\boldsymbol{V}}$ with respect to the iterate $k$ associated to the algorithms 2.22, 2.22 with fixed step $\lambda_{k}=1$ and CG respectively. We also consider the so-called by analogy damped quasi newton method

$$
\left\{\begin{array}{l}
y_{0} \in \boldsymbol{H} \quad \text { given, } \\
y_{k+1}=y_{k}-\lambda_{k} \widetilde{Y}_{1, k}, \quad k \geq 0 \\
\lambda_{k}=\operatorname{argmin}_{\lambda \in[0, m]} E\left(y_{k}-\lambda \widetilde{Y}_{1, k}\right)
\end{array}\right.
$$

with $\widetilde{Y}_{1, k} \in \boldsymbol{V}$ the solution of the formulation

$\alpha \int_{\Omega} \tilde{Y}_{1, k} \cdot w+\nu \int_{\Omega} \nabla \tilde{Y}_{1, k} \cdot \nabla w+\int_{\Omega}\left(y_{0} \cdot \nabla \tilde{Y}_{1, k}+\tilde{Y}_{1, k} \cdot \nabla y_{0}\right) \cdot w=-\alpha \int_{\Omega} v_{k} \cdot w-\nu \int_{\Omega} \nabla v_{k} \cdot \nabla w, \forall w \in \boldsymbol{V}$ and $v_{k} \in \boldsymbol{V}$ the corrector (associated to $y_{k}$ ) solution of 2.8 .

A regular mesh composed of 14143 triangles and 7360 vertices depicted in Figure 2 is used. Table 2 reports the evolution of the norm of the corrector $\left\|v_{k}\right\|_{\boldsymbol{V}}=\sqrt{2 E\left(y_{k}\right)}$, an upper bound of $\left\|y-y_{k}\right\|_{\boldsymbol{V}}$, according to Proposition 2.8. As expected in view of the results in Section 2.2. the descent algorithm 2.22 based on $Y_{1, k}$ is much faster than the CG algorithm. Moreover, the optimal values for the optimal step $\lambda_{k}$ are close to one, so that the Newton method provides a similar speed of convergence. As the norm of $Y_{1, k}$ goes to zero with $k$, the term factor of $\lambda^{2}$ 
in (2.24) gets small, and the optimal $\lambda_{k}$ gets close to one. Remark as well that the algorithm 4.1), whose each iteration involves the same matrice to be inverted, offers an excellent speed of convergence. In term of CPU times, Algorithm (2.22) and (4.1) require about 53 and 108 seconds respectively and leads to the same approximation. We have notably $\|\nabla \cdot y\|_{L^{2}(\Omega)}=1.59 \times 10^{-2}$ and $\|\nabla \cdot y\|_{L^{2}(\Omega)} /|\Omega|=1.83 \times 10^{-4}$.

\begin{tabular}{|c|c|c|c|c|}
\hline$\sharp$ iterate $k$ & 2.22 with $\lambda_{k}=1$ & 2.22 & (4.1) & $\mathrm{CG}$ \\
\hline 1 & $4.442 \times 10^{-1}$ & $3.798 \times 10^{-1}(0.8545)$ & $3.796 \times 10^{-1}$ & $5.214 \times 10^{-2}$ \\
\hline 2 & $1.959 \times 10^{-1}$ & $1.810 \times 10^{-1}(0.9573)$ & $1.592 \times 10^{-1}$ & $4.195 \times 10^{-2}$ \\
\hline 3 & $5.609 \times 10^{-2}$ & $4.045 \times 10^{-2}(0.9949)$ & $4.375 \times 10^{-2}$ & $3.276 \times 10^{-2}$ \\
\hline 4 & $3.986 \times 10^{-3}$ & $2.223 \times 10^{-3}(1.0006)$ & $6.055 \times 10^{-3}$ & $2.946 \times 10^{-2}$ \\
\hline 5 & $2.082 \times 10^{-5}$ & $5.719 \times 10^{-6}(0.9999)$ & $6.808 \times 10^{-3}$ & $2.568 \times 10^{-2}$ \\
\hline 6 & $5.912 \times 10^{-10}$ & $4.959 \times 10^{-11}(1)$ & $9.899 \times 10^{-4}$ & $2.290 \times 10^{-2}$ \\
\hline 7 & $4.881 \times 10^{-15}$ & $3.299 \times 10^{-15}(1)$ & $9.009 \times 10^{-4}$ & $2.219 \times 10^{-2}$ \\
\hline 8 & - & - & $1.486 \times 10^{-4}$ & $2.024 \times 10^{-2}$ \\
\hline 9 & - & - & $9.553 \times 10^{-5}$ & $1.952 \times 10^{-2}$ \\
\hline 10 & - & - & $2.092 \times 10^{-5}$ & $1.819 \times 10^{-2}$ \\
\hline 11 & - & - & $1.396 \times 10^{-5}$ & $1.764 \times 10^{-2}$ \\
\hline 12 & - & - & $3.170 \times 10^{-6}$ & $1.723 \times 10^{-2}$ \\
\hline 13 & - & - & $1.839 \times 10^{-6}$ & $1.674 \times 10^{-2}$ \\
\hline 14 & - & - & $3.809 \times 10^{-7}$ & $1.657 \times 10^{-2}$ \\
\hline 15 & - & - & $1.987 \times 10^{-7}$ & $1.606 \times 10^{-2}$ \\
\hline 26 & - & - & $4.321 \times 10^{-13}$ & $1.120 \times 10^{-2}$ \\
\hline 200 & - & - & - & $2.091 \times 10^{-5}$ \\
\hline
\end{tabular}

TABLE 1. 2D channel geometry; $\nu=1 / 150$; Evolution of $\left\|y_{k+1}-y_{k}\right\|_{V} /\left\|y_{k}\right\|_{V}$ with respect to $k$.

\begin{tabular}{|c|c|c|c|c|}
\hline$\sharp$ iterate $k$ & 2.22 with $\lambda_{k}=1$ & 2.22 & 4.1 & CG \\
\hline 1 & $5.467 \times 10^{-2}$ & $5.467 \times 10^{-2}$ & $5.476 \times 10^{-2}$ & $5.467 \times 10^{-2}$ \\
\hline 2 & $2.398 \times 10^{-2}$ & $2.224 \times 10^{-2}$ & $2.222 \times 10^{-2}$ & $3.701 \times 10^{-2}$ \\
\hline 3 & $4.953 \times 10^{-3}$ & $4.601 \times 10^{-3}$ & $5.457 \times 10^{-3}$ & $2.917 \times 10^{-2}$ \\
\hline 4 & $3.201 \times 10^{-4}$ & $1.565 \times 10^{-4}$ & $9.322 \times 10^{-4}$ & $2.492 \times 10^{-2}$ \\
\hline 5 & $1.530 \times 10^{-6}$ & $5.437 \times 10^{-7}$ & $5.191 \times 10^{-4}$ & $2.201 \times 10^{-2}$ \\
\hline 6 & $3.650 \times 10^{-11}$ & $4.227 \times 10^{-12}$ & $1.712 \times 10^{-4}$ & $1.995 \times 10^{-2}$ \\
\hline 7 & $6.541 \times 10^{-16}$ & $2.541 \times 10^{-16}$ & $1.712 \times 10^{-4}$ & $1.840 \times 10^{-2}$ \\
\hline 8 & - & - & $7.852 \times 10^{-5}$ & $1.709 \times 10^{-2}$ \\
\hline 9 & - & - & $2.472 \times 10^{-5}$ & $1.603 \times 10^{-2}$ \\
\hline 10 & - & - & $8.953 \times 10^{-6}$ & $1.511 \times 10^{-2}$ \\
\hline 11 & - & - & $3.424 \times 10^{-6}$ & $1.433 \times 10^{-2}$ \\
\hline 12 & - & - & $1.205 \times 10^{-6}$ & $1.363 \times 10^{-2}$ \\
\hline 13 & - & - & $4.251 \times 10^{-7}$ & $1.301 \times 10^{-2}$ \\
\hline 14 & - & - & $1.366 \times 10^{-7}$ & $1.242 \times 10^{-2}$ \\
\hline 15 & - & - & $4.478 \times 10^{-8}$ & $1.187 \times 10^{-2}$ \\
\hline 26 & - & - & $1.599 \times 10^{-14}$ & $6.259 \times 10^{-3}$ \\
\hline 200 & - & - & - & $1.551 \times 10^{-5}$ \\
\hline
\end{tabular}

TABLE 2. 2D channel geometry; $\nu=1 / 150 ;$ Evolution of $\left\|v_{k}\right\|_{\boldsymbol{V}}=\sqrt{2 E\left(y_{k}\right)}$ with respect to $k$. 
For smaller values of $\nu$, the results are qualitatively differents. Table 3 reports some norms with respect to $k$ for $\nu=1 / 700$. We observe, from the last column, that the Newton method for which $\lambda_{k}$ is fixed to one does not converge anymore. Actually, the Newton method, when initialized with the solution of the corresponding Stokes problem, diverges for $\nu \leq 1 / 250$. On the other hand, the optimization of the step $\lambda_{k}$ produces a very fast convergence of the sequence $\left\{y_{k}\right\}_{(k>0)}$. Observe here that the values for the optimal $\lambda_{k}$ are not close to one, during the first iterations. We obtain notably $\|\nabla \cdot y\|_{L^{2}(\Omega)} /|\Omega|=5.78 \times 10^{-2}$. In agreement with Theorem 2.15 . we observe from Table 3 that the decrease of $\sqrt{E\left(y_{k}\right)}$ to zero is first linear and then becomes quadratic.

The algorithm 4.1 is a bit more robust than the Newton one as it converges for all $\nu$ satisfying $\nu \geq 1 / 290$ approximately. Finally, as discussed in [11], the CG algorithm converges and produces similar numerical values: the convergence is however much slower since about 350 iterates are needed to achieve $\sqrt{2 E\left(y_{k}\right)}$ of the order $10^{-3}$.

The algorithm 2.22 requires however the initial guess to be close enough to the solution. Initialized with the solution of the corresponding Stokes problem, it diverges for $\nu \leq 1 / 720$. A continuation method with respect to $\nu$ is then necessary in that case. Algorithm 2.22 is also robust with respect to the mesh size: with a twice finer mesh composed of 84707 triangles and 43069 vertices, the convergence $\left\|y^{k+1}-y^{k}\right\| \leq 10^{-12}\left\|y^{k}\right\|$ is observed after $k=18$ iterates (instead of 14 for the previous coarser mesh) leading notably to $\|\nabla \cdot y\|_{L^{2}(\Omega)} /|\Omega|=3.91 \times 10^{-2}$. Figure 3 depicts the streamlines of the convergent sequence $y_{k}$ in the cases $\nu=1 / 150$ and $\nu=1 / 700$. The method allows to capture the shear layer developing in the flow behind the re-entrant corner.

\begin{tabular}{|c|c|c|c||c|}
\hline$\sharp$ iterate $k$ & $\left\|y_{k+1}-y_{k}\right\|_{V} /\left\|y_{k}\right\|_{V}$ & $\sqrt{2 E\left(y_{k}\right)}$ & $\lambda_{k}$ & $\sqrt{2 E\left(y_{k}\right)}$ with $\lambda_{k}=1$ \\
\hline 1 & $7.153 \times 10^{-1}$ & $5.467 \times 10^{-2}$ & 0.727 & $5.467 \times 10^{-2}$ \\
2 & $1.424 \times 10^{-4}$ & $2.791 \times 10^{-2}$ & $4.77 \times 10^{-5}$ & $3.452 \times 10^{-2}$ \\
3 & $2.073 \times 10^{-1}$ & $2.791 \times 10^{-2}$ & $2.01 \times 10^{-2}$ & $8.089 \times 10^{-2}$ \\
4 & $3.538 \times 10^{-1}$ & $2.737 \times 10^{-2}$ & 0.958 & $5.344 \times 10^{-2}$ \\
5 & $9.138 \times 10^{-2}$ & $7.270 \times 10^{-3}$ & $4.81 \times 10^{-6}$ & 2.409 \\
6 & $6.244 \times 10^{-2}$ & $2.622 \times 10^{-3}$ & $1.73 \times 10^{-3}$ & $6.115 \times 10^{-1}$ \\
7 & $2.028 \times 10^{-2}$ & $1.078 \times 10^{-3}$ & 0.358 & 3.944 \\
8 & $3.695 \times 10^{-3}$ & $2.610 \times 10^{-4}$ & 0.521 & $9.851 \times 10^{1}$ \\
9 & $7.522 \times 10^{-4}$ & $4.184 \times 10^{-5}$ & 1.098 & $8.186 \times 10^{1}$ \\
10 & $9.886 \times 10^{-6}$ & $6.014 \times 10^{-7}$ & 0.963 & $4.385 \times 10^{4}$ \\
11 & $3.872 \times 10^{-6}$ & $1.692 \times 10^{-7}$ & 1.032 & $1.093 \times 10^{4}$ \\
12 & $6.820 \times 10^{-11}$ & $4.404 \times 10^{-12}$ & 0.9983 & $3.169 \times 10^{4}$ \\
13 & $1.288 \times 10^{-10}$ & $2.880 \times 10^{-12}$ & 0.9999 & $1.576 \times 10^{5}$ \\
14 & $6.879 \times 10^{-15}$ & $3.263 \times 10^{-16}$ & 1. & $4.068 \times 10^{4}$ \\
\hline
\end{tabular}

TABLE 3. 2D channel geometry; $\nu=1 / 700$; Results for the algorithm (2.22).

4.2. Steady case: 2D semi-circular cavity. We now consider the test discussed in [5]. The geometry is a semi-disk $\Omega=\left\{\left(x_{1}, x_{2}\right) \in \mathbb{R}^{2}, x_{1}^{2}+x_{2}^{2}<1 / 4, x_{2} \leq 0\right\}$ depicted on Figure 4 . The velocity is imposed to $y=(g, 0)$ on $\Gamma_{0}=\left\{\left(x_{1}, 0\right) \in \mathbb{R}^{2},\left|x_{1}\right|<1 / 2\right\}$ with $g$ vanishing at $x_{1}= \pm 1 / 2$ and close to one elsewhere: we take $g\left(x_{1}\right)=\left(1-e^{100\left(x_{1}-1 / 2\right)}\right)\left(1-e^{-100\left(x_{1}+1 / 2\right)}\right)$. On the rest $\Gamma_{1}=\left\{\left(x_{1}, x_{2}\right) \in \mathbb{R}^{2}, x_{2}<0, x_{1}^{2}+x_{2}^{2}=1 / 4\right\}$ of the boundary the velocity is fixed to zero.

For a regular triangular mesh, composed of 79628 triangles and 40205 vertices, leading to a mesh size $h \approx 6.23 \times 10^{-3}$, the Newton method $\left(\lambda_{k}=1\right)$ initialized with the corresponding Stokes solution, converges up to $\nu^{-1} \approx 500$. On the other hand, the algorithm 2.22 still converges 


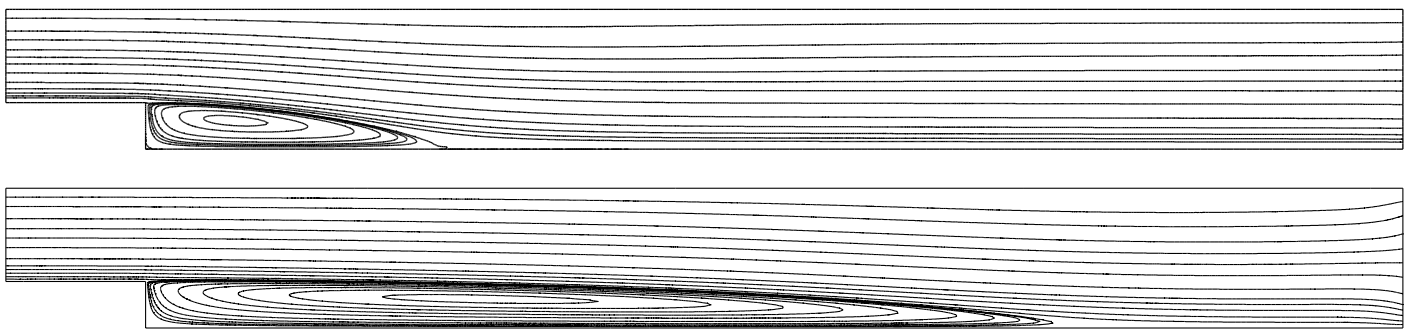

Figure 3. Streamlines of the steady state solution at $\nu=1 / 150$ (top) and $\nu=1 / 700$ (bottom); $L=30$.

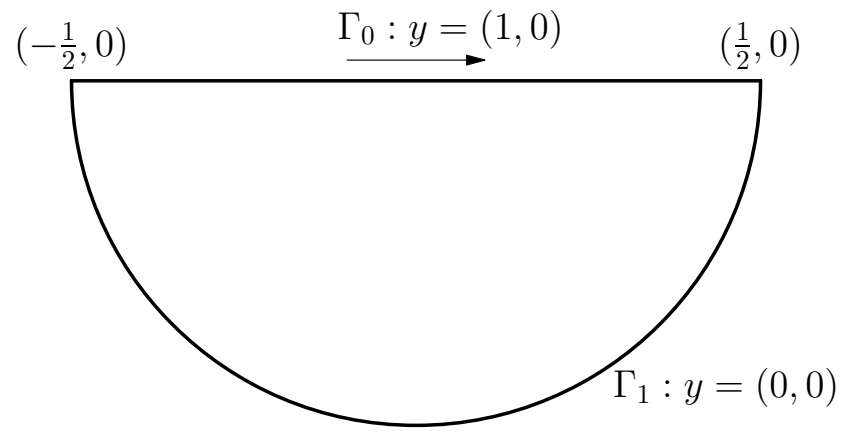

FiguRE 4. Semi-disk geometry.

up to $\nu^{-1} \approx 910$. Figures 7 depicts the streamlines of the steady state solution corresponding to $\nu^{-1}=500$ and to $\nu^{-1}=i \times 10^{3}$ for $i=1, \cdots, 9$. The figures are in very good agreements with those depicted in [5]. The solution corresponding to $\nu^{-1}=500$ is obtained from the sequence given (2.22) initialized with the Stokes solution. Eight iterates are necessary to achieve $\sqrt{2 E(y)} \approx 3.4 \times 10^{-17}$. The stopping criterion is $\left\|y_{k+1}-y_{k}\right\|_{\boldsymbol{V}} \leq 10^{-12}\left\|y_{k}\right\|_{\boldsymbol{V}}$. Tables 4 and 5 collects some values for $\nu=1 / 500$ and $\nu=1 / 700$. Then, the other solutions are obtained by a continuation method with respect to $\nu$ taking $\delta \nu^{-1}=500$. For instance, the solution corresponding to $\nu^{-1}=5000$ is obtained from the algorithm 2.22 initialized with the steady solution corresponding to $\nu^{-1}=4500$. Table 8 reports the history of the continuation method and highlights the efficiency of the algorithm (2.22): up to $\nu^{-1}=9500$, few iterations achieve the convergence of the minimizing sequence $\left\{y_{k}\right\}_{k \in \mathbb{N}}$. From $\nu^{-1}=10^{4}$, with a finer mesh (for which the mesh size is $h \approx 4.37 \times 10^{-3}$ ), $\delta \nu$ is reduced to $\delta \nu^{-1}=100$ and leads to convergence beyond $\nu^{-1}=15000$. Table also reports the minimal value of the streamline function $\psi$ which compare very well with those of [5].

\begin{tabular}{|c|c|c|c||c|}
\hline$\sharp$ iterate $k$ & $\left\|y_{k+1}-y_{k}\right\|_{V} /\left\|y_{k}\right\|_{V}$ & $\sqrt{2 E\left(y_{k}\right)}$ & $\lambda_{k}$ & $\sqrt{2 E\left(y_{k}\right)}$ with $\lambda_{k}=1$ \\
\hline 1 & $4.66 \times 10^{-1}$ & $8.51 \times 10^{-3}$ & 0.810 & $8.51 \times 10^{-3}$ \\
2 & $2.03 \times 10^{-1}$ & $3.57 \times 10^{-3}$ & 0.716 & $4.09 \times 10^{-3}$ \\
3 & $9.54 \times 10^{-2}$ & $1.36 \times 10^{-3}$ & 0.860 & $2.26 \times 10^{-3}$ \\
4 & $2.64 \times 10^{-2}$ & $3.38 \times 10^{-4}$ & 0.982 & $2.51 \times 10^{-4}$ \\
5 & $1.53 \times 10^{-3}$ & $2.43 \times 10^{-5}$ & 1.002 & $3.47 \times 10^{-6}$ \\
6 & $4.21 \times 10^{-6}$ & $6.36 \times 10^{-8}$ & 1. & $1.54 \times 10^{-9}$ \\
7 & $4.20 \times 10^{-11}$ & $6.50 \times 10^{-13}$ & 1. & $1.52 \times 10^{-16}$ \\
8 & $3.25 \times 10^{-15}$ & $3.45 \times 10^{-17}$ & 1. & $3.39 \times 10^{-17}$ \\
\hline
\end{tabular}

TABLE 4. Semi-disk geometry ; $\nu=1 / 500$; Results for the algorithm $(2.22)$. 


\begin{tabular}{|c|c|c|c||c|}
\hline$\sharp$ iterate $k$ & $\left\|y_{k+1}-y_{k}\right\|_{V} /\left\|y_{k}\right\|_{V}$ & $\sqrt{2 E\left(y_{k}\right)}$ & $\lambda_{k}$ & $\sqrt{2 E\left(y_{k}\right)}$ with $\lambda_{k}=1$ \\
\hline 1 & $4.89 \times 10^{-1}$ & $8.51 \times 10^{-3}$ & 0.702 & $8.51 \times 10^{-3}$ \\
2 & $2.41 \times 10^{-1}$ & $4.43 \times 10^{-3}$ & 0.583 & $5.75 \times 10^{-3}$ \\
3 & $1.48 \times 10^{-1}$ & $2.15 \times 10^{-3}$ & 0.510 & $5.27 \times 10^{-3}$ \\
4 & $1.01 \times 10^{-1}$ & $1.15 \times 10^{-3}$ & 0.568 & $1.23 \times 10^{-2}$ \\
5 & $6.83 \times 10^{-2}$ & $6.50 \times 10^{-4}$ & 0.931 & $4.40 \times 10^{-3}$ \\
6 & $1.19 \times 10^{-2}$ & $1.76 \times 10^{-4}$ & 1.018 & $1.52 \times 10^{-2}$ \\
7 & $3.35 \times 10^{-4}$ & $3.42 \times 10^{-6}$ & 0.999 & $5.06 \times 10^{-3}$ \\
8 & $3.19 \times 10^{-7}$ & $4.22 \times 10^{-9}$ & 1. & $1.45 \times 10^{-2}$ \\
9 & $2.97 \times 10^{-13}$ & $2.30 \times 10^{-15}$ & 1. & $1.85 \times 10^{-2}$ \\
20 & - & - & - & 5.39 \\
\hline
\end{tabular}

TABLE 5. Semi-disk geometry ; $\nu=1 / 700 ;$ Results for the algorithm $(2.22)$.
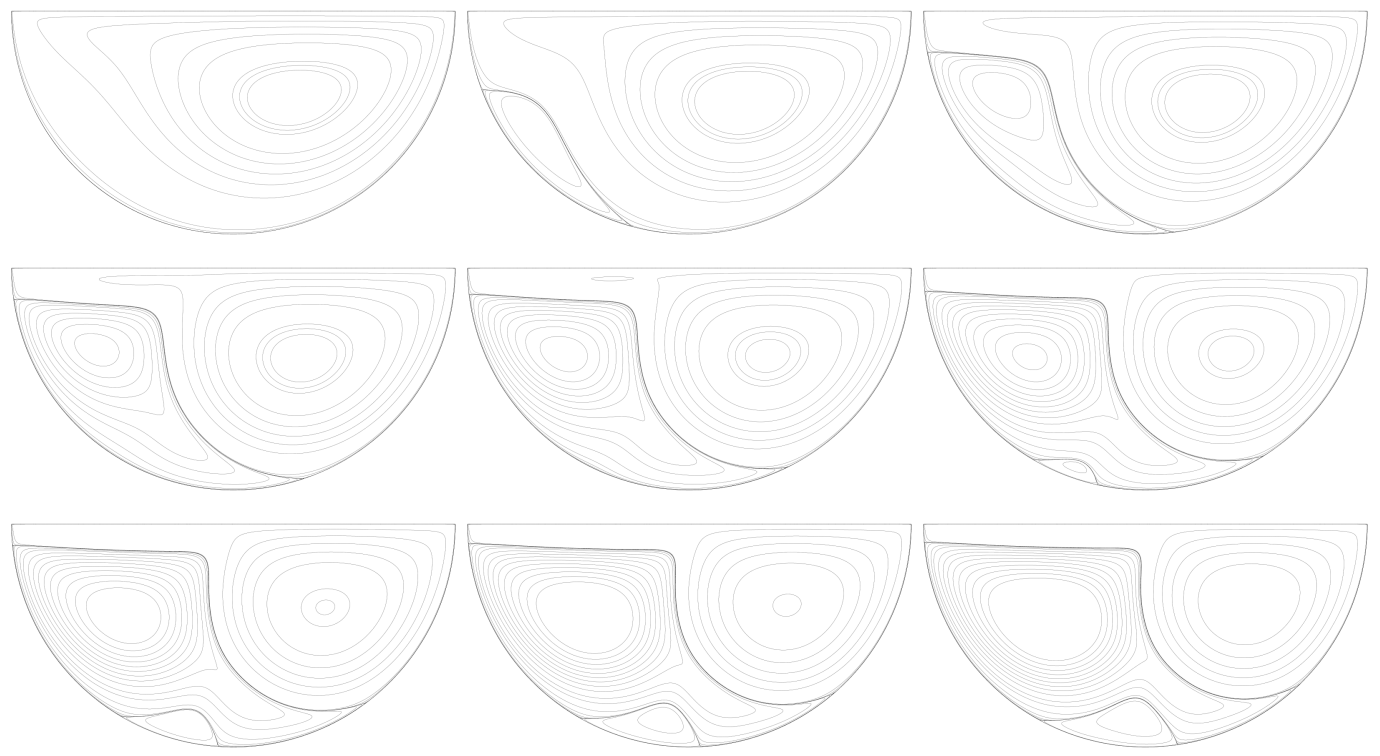

Figure 5. Streamlines of the steady state solution for $\nu^{-1}=$ $500,1000,2000,3000,4000,5000,6000,7000$ and $\nu^{-1}=8000$.

The case $\alpha>0$ leads to similar results, in full agreement with the theoretical Section 2 . For $\nu=1 / 1000$, Table 6 reports results of the algorithm 2.22 for $\alpha \in\left\{10^{-1}, 1 ., 10 ., 100.\right\}$. As expected, the gain of coercivity of the functional $E$ involves a notable robustness and speed up of the algorithm. Recall that for $\alpha=0$. and $\nu=1 / 1000$, algorithm 2.22 does not converge. For $\alpha=10^{-1}$, we observe the convergence after 8 iterates. For a fixed value of $\nu$, this number of iterates decreases as $\alpha$ gets larger. Actually, we observe that when $\alpha \sqrt{\nu}=\mathcal{O}(1)$, algorithm 2.22 converges after few iterates with $\lambda_{k}$ close to 1 for all $k$. Moreover, when $\alpha \nu=\mathcal{O}(1)$, the convergence is achieved after one iterate only. This behavior suggests that one may recover the solution of the steady Navier-Stokes system (corresponding to $\alpha=0$ ) by using a continuation procedure with respect to the parameter $\alpha$ decreasing to zero. We easily check, for any $\alpha \geq 0$, the estimate $\left\|\nabla\left(y_{\alpha}-y_{\alpha=0}\right)\right\|_{2} \leq c_{p} \sqrt{\frac{\alpha}{\nu}}\left\|y_{\alpha=0}\right\|_{2}$ where $y_{\alpha}$ solves $(1.5)$, and $c_{p}$ the Poincaré constant. For $\nu=1 / 5000$, Table 7 reports the history of the continuation approach starting from $\alpha=1$. to $\alpha=0$ with intermediate step $\alpha \in\left\{10^{-1}, 10^{-2}, 10^{-3}, 10^{-4}\right\}$. Figure 6 depicts the evolution of the sequences $\left\{\sqrt{2 E\left(y_{k}\right)}\right\}_{k}$ and $\left\{\lambda_{k}\right\}_{k}$ obtained from the algorithm 2.22 with $\alpha=10^{-3}$. The algorithm is initialized with the solution corresponding to $\alpha=10^{-2}$. The behavior of these 
sequence fully illustrates Theorem 2.15 and the robustness of the method: as $\lambda_{k}$ increases, the decay of $\sqrt{2 E\left(y_{k}\right)}$, initially low, gets larger and becomes very fast when $\lambda_{k}$ is close to one. Figure 7 reports the streamlines of the solution for various values of $\alpha$.

\begin{tabular}{|c|c|c|c|c|}
$\#$ iterate $k$ & $\alpha=0.1$ & $\alpha=1$. & $\alpha=10$. & $\alpha=100$. \\
\hline 1 & $6.59 \times 10^{-3}(0.65)$ & $3.11 \times 10^{-3}(0.87)$ & $1.04 \times 10^{-3}(0.99)$ & $2.99 \times 10^{-4}(9.99)$ \\
2 & $3.54 \times 10^{-3}(0.63)$ & $7.59 \times 10^{-4}(0.98)$ & $3.68 \times 10^{-5}(0.99)$ & $7.81 \times 10^{-7}(1)$. \\
3 & $1.55 \times 10^{-3}(0.64)$ & $5.94 \times 10^{-5}(1.00)$ & $5.15 \times 10^{-8}(1)$. & $6.92 \times 10^{-12}(1)$. \\
4 & $7.05 \times 10^{-4}(0.74)$ & $4.84 \times 10^{-7}(1)$. & $1.20 \times 10^{-13}$ & $5.50 \times 10^{-17}$ \\
5 & $2.76 \times 10^{-4}(1.01)$ & $2.63 \times 10^{-11}(1)$. & - & - \\
6 & $2.95 \times 10^{-5}(1)$. & $1.03 \times 10^{-17}$ & - & - \\
7 & $1.55 \times 10^{-7}(1)$. & - & - & - \\
8 & $1.99 \times 10^{-11}(1)$. & - & - & - \\
9 & $1.60 \times 10^{-17}$ & - & - & - \\
\hline
\end{tabular}

TABLE 6. Semi-disk geometry ; $\nu=1 / 1000$; Results for the algorithm 2.22); $\sqrt{2 E\left(y_{k}\right)}$ and $\lambda_{k}$ with respect to $\alpha$.

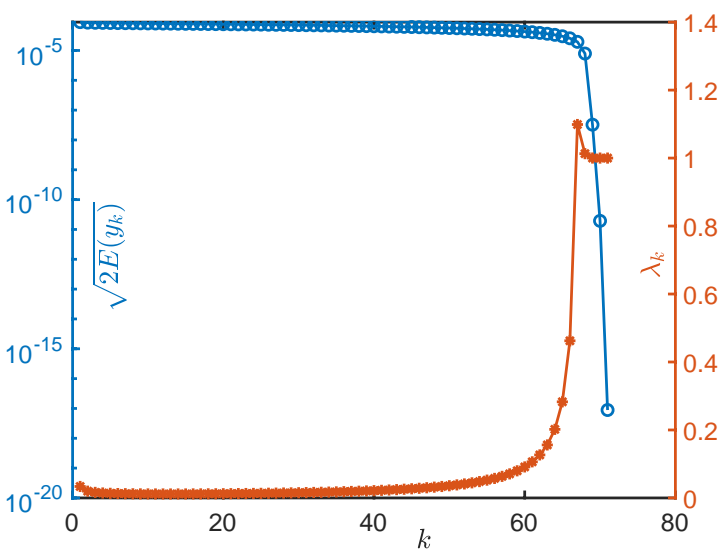

Figure 6. Semi-disk geometry; $\nu=1 / 5000$; Evolution of $\sqrt{2 E\left(y_{k}\right)}$ and $\lambda_{k}$ w.r.t. $k$ for $\alpha$ from $10^{-2}$ to $10^{-3}$.
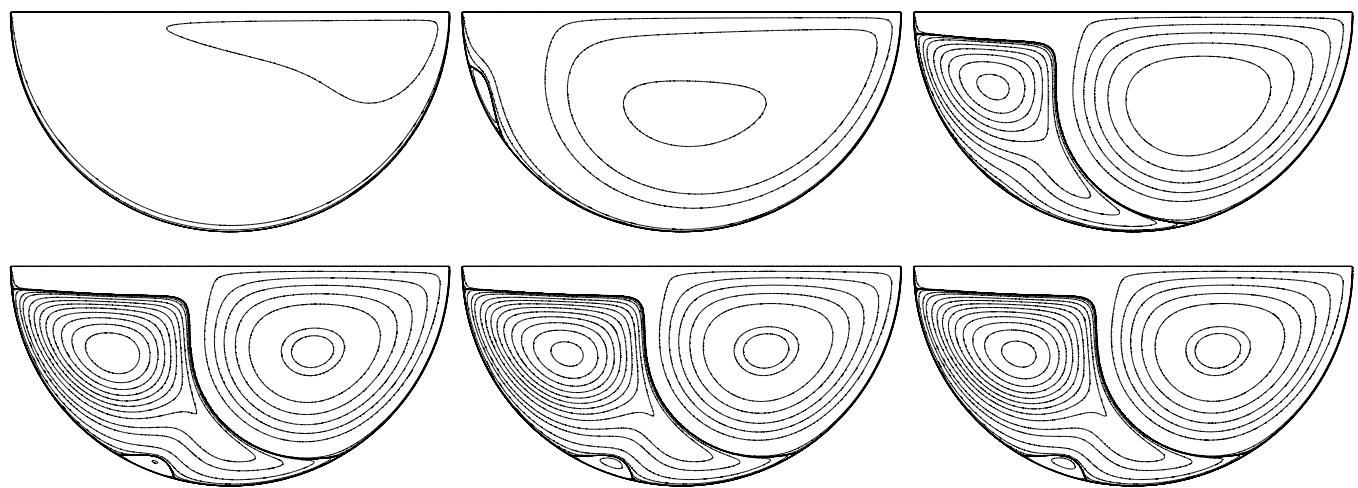

Figure 7 . Streamlines of the $\alpha$-steady state solution for $\alpha=1 ., 10^{-1}, 10^{-2}$ (Top) and $\alpha=10^{-3}, 10^{-4}$ and 0 . (Bottom). 


\begin{tabular}{|c|cccc|}
\hline$\alpha$ & $\sharp$ it. & $\left\|\pi_{\alpha}\right\|_{L^{2}(\Omega)}$ & $\left\|y_{\alpha}\right\|_{\boldsymbol{V}}$ & $\left\|y_{\alpha}-y_{\alpha=0}\right\|_{\boldsymbol{V}}$ \\
\hline Stokes $\rightarrow 1$. & 6 & 0.00684 & 7.659 & 4.28 \\
$1 . \rightarrow 10^{-1}$ & 37 & 0.011 & 7.4896 & 3.32 \\
$10^{-1} \rightarrow 10^{-2}$ & 49 & 0.0297 & 7.8951 & 2.67 \\
$10^{-2} \rightarrow 10^{-3}$ & 70 & 0.0360 & 8.0166 & $4.02 \times 10^{-1}$ \\
$10^{-3} \rightarrow 10^{-4}$ & 12 & 0.0366 & 8.0278 & $4.03 \times 10^{-2}$ \\
$10^{-4} \rightarrow 0$ & 4 & 0.0367 & 8.029 & $4.03 \times 10^{-3}$ \\
\hline
\end{tabular}

TABle 7. Semi-disk geometry; Continuation method with respect to $\alpha$ for the solution of the steady Navier-Stokes equation; $\nu=1 / 5000$.

\begin{tabular}{|c|c|c|c|c|c|c|}
\hline$\nu^{-1}$ & $\sharp$ it. & $\|\pi\|_{L^{2}(\Omega)}$ & $\left|y_{1}\right|_{H_{0}^{1}(\Omega)}$ & $\left|y_{2}\right|_{H_{0}^{1}(\Omega)}$ & $\min _{\Omega} \psi$ & $\min _{\Omega} \psi[5$ \\
\hline Stokes $\rightarrow 500$ & 7 & $4.31 \times 10^{-2}$ & 4.462 & 2.489 & -0.0766784 & - \\
\hline $500 \rightarrow 1000$ & 7 & $4.07 \times 10^{-2}$ & 4.919 & 2.883 & -0.0780642 & -0.0779 \\
\hline $1000 \rightarrow 1500$ & 6 & 0.0399 & 5.2966 & 3.15371 & -0.0775772 & - \\
\hline $1500 \rightarrow 2000$ & 6 & 0.0393087 & 5.61222 & 3.36132 & -0.0766604 & -0.0763 \\
\hline $2000 \rightarrow 2500$ & 5 & 0.0388207 & 5.8849 & 3.53141 & -0.0756008 & - \\
\hline $2500 \rightarrow 3000$ & 5 & 0.0383734 & 6.12689 & 3.67571 & -0.074476 & -0.0742 \\
\hline $3000 \rightarrow 3500$ & 5 & 0.0379483 & 6.34559 & 3.80097 & -0.0733293 & - \\
\hline $3500 \rightarrow 4000$ & 5 & 0.0375405 & 6.54581 & 3.91156 & -0.0721912 & - \\
\hline $4000 \rightarrow 4500$ & 6 & 0.0371478 & 6.73091 & 4.01047 & -0.0710789 & - \\
\hline $4500 \rightarrow 5000$ & 6 & 0.0367688 & 6.90338 & 4.09986 & -0.0699992 & -0.0700 \\
\hline $5000 \rightarrow 5500$ & 6 & 0.0364024 & 7.06514 & 4.18132 & -0.0689569 & - \\
\hline $5500 \rightarrow 6000$ & 6 & 0.0360479 & 7.21768 & 4.25607 & -0.0679526 & - \\
\hline $6000 \rightarrow 6500$ & 6 & 0.0357043 & 7.3622 & 4.32506 & -0.0669879 & - \\
\hline $6500 \rightarrow 7000$ & 6 & 0.0353712 & 7.49966 & 4.38908 & -0.0660596 & - \\
\hline $7000 \rightarrow 7500$ & 5 & 0.0350479 & 7.63085 & 4.44875 & -0.0651669 & - \\
\hline $7500 \rightarrow 8000$ & 5 & 0.0347341 & 7.75643 & 4.5046 & -0.0643104 & - \\
\hline $8000 \rightarrow 8500$ & 6 & 0.0344295 & 7.87694 & 4.55705 & -0.0634864 & - \\
\hline $8500 \rightarrow 9000$ & 6 & 0.0341339 & 7.99287 & 4.60649 & -0.0626943 & - \\
\hline $9000 \rightarrow 9500$ & 11 & 0.0338468 & 8.10461 & 4.65324 & -0.0619334 & - \\
\hline $9500 \rightarrow 10000$ & 39 & 0.0335673 & 8.21355 & 4.6937 & -0.061204 & - \\
\hline $10000 \rightarrow 10100$ & 5 & 0.0335126 & 8.23472 & 4.70226 & -0.061061 & - \\
\hline $10100 \rightarrow 10200$ & 5 & 0.0334581 & 8.25574 & 4.71073 & -0.0609195 & - \\
\hline $10200 \rightarrow 10300$ & 5 & 0.033404 & 8.27663 & 4.71911 & -0.0607786 & - \\
\hline $10300 \rightarrow 10400$ & 5 & 0.0333502 & 8.29738 & 4.72741 & -0.0606386 & - \\
\hline $10400 \rightarrow 10500$ & 5 & 0.0332967 & 8.318 & 4.73563 & -0.0605006 & - \\
\hline
\end{tabular}

TABLE 8. Semi-disk geometry; Continuation method with respect to $\nu$ for the solution of the steady Navier-Stokes equation; $\alpha=0$.

4.3. Unsteady case: 2D semi-circular cavity. We now use the least-squares method in order to solve iteratively the implicit Euler scheme (1.2). The parameter $\alpha=1 / \delta t$ is strictly positive. We remind that for $\nu$ approximatively larger than $1 / 6600$, the unsteady solution converges as time evolves to the steady solution (corresponding to $\alpha=0$ ) obtained in the previous section by continuation technics. Actually, the iterative process due the time discretization can also be seen as a continuation approach. We consider the value $\nu=1 / 1000$. Following [5], we take as initial condition $u_{0}$ the steady-state solution corresponding to $\nu=1 / 500$. For the value $\alpha=200$ corresponding to the time discretization parameter $\delta t=5 \times 10^{-3}$, we observe the convergence of the sequence $\left\{y_{k}^{n+1}\right\}_{k>0}$ after at most three iterations, for each $n$ (except for 
$n=0$ requiring 6 iterations). For the value $\alpha=2000$ corresponding to $\delta t=5 \times 10^{-4}$ (used in [5]), we observe the convergence of the sequence after one iterate. At time $T=10$, the unsteady state solution is close to the solution of the steady Navier-Stokes equation: we compute that the sequence $\left\{y^{n}\right\}_{n=0, \ldots, 2000}$ satisfies $\left\|y^{2000}-y^{1999}\right\|_{L^{2}(\Omega)} /\left\|y^{2000}\right\|_{L^{2}(\Omega)} \approx 1.19 \times 10^{-5} \cdot n=2000$ corresponds to $T=2000 \times \delta t=10$. Figures 8 display the streamlines of the unsteady state solution corresponding to $\nu=1 / 1000$ at time $0,1,2,3,4,5,6$ and 7 seconds to be compared with the streamlines of the steady solution depicted in Figure 7 . These figures are in full agreement with [5.
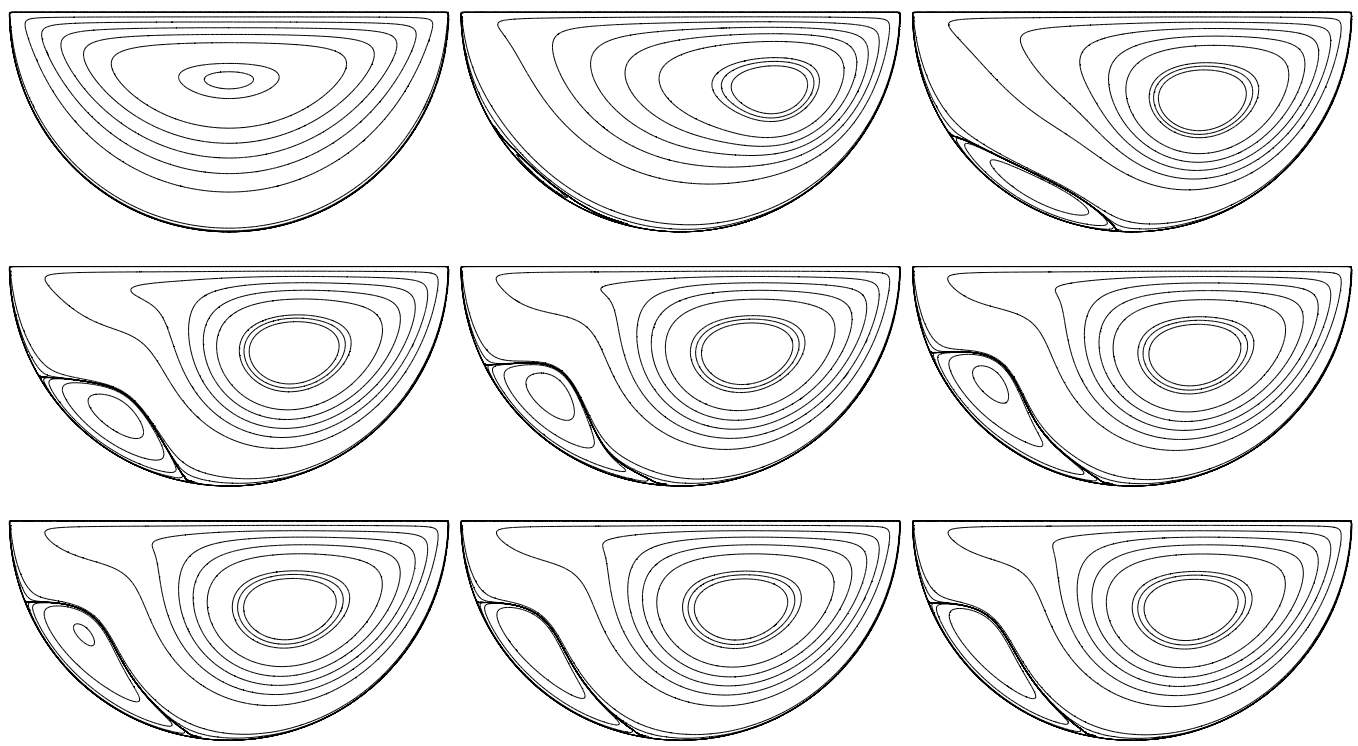

FiguRE 8 . Streamlines of the unsteady state solution for $\nu^{-1}=1000$ at time $t=i, i=0, \cdots, 8 \mathrm{~s}$.

Before to compare with standard time marching schemes, we emphasize that, for each $n$, algorithm 3.4 reads as follows

$$
\left\{\begin{array}{l}
y_{0}^{n+1}=y^{n} \\
\int_{\Omega} \alpha y_{k+1}^{n+1} \cdot w+\nu \nabla y_{k+1}^{n+1} \cdot \nabla w+y_{k}^{n+1} \cdot \nabla y_{k+1}^{n+1} \cdot w+y_{k+1}^{n+1} \cdot \nabla y_{k}^{n+1} \cdot w= \\
\left(1-\lambda_{k}\right) \int_{\Omega} \alpha y_{k}^{n+1} \cdot w+\nu \nabla y_{k}^{n+1} \cdot \nabla w+y_{k}^{n+1} \cdot \nabla y_{k}^{n+1} \cdot w \\
+\lambda_{k} \int_{\Omega} \alpha y^{n} \cdot w+<f^{n}, w>_{H^{-1}(\Omega), H_{0}^{1}(\Omega)}+\int_{\Omega} y_{k}^{n+1} \cdot \nabla y_{k}^{n+1} \cdot w \quad k \geq 0, \forall w \in \boldsymbol{V}
\end{array}\right.
$$

where the optimal step $\lambda_{k}$ minimize $\lambda \rightarrow E_{n}\left(y_{k}^{n+1}-\lambda Y_{1, k}^{n+1}\right)$. For $\alpha$ large, the optimal step $\lambda_{k}$ equals one and the convergence with respect to $k$ is achieved after one iterate. The convergent approximation $y^{n+1}:=y_{1}^{n+1}$ then simply solves, for each $n$ the following semi-implicit scheme (mentioned in [15, section 13.4])

$$
\begin{aligned}
& \int_{\Omega} \alpha y^{n+1} \cdot w+\nu \nabla y^{n+1} \cdot \nabla w+y^{n} \cdot \nabla y^{n+1} \cdot w+y^{n+1} \cdot \nabla y^{n} \cdot w \\
& \quad=\int_{\Omega} \alpha y^{n} \cdot w+<f^{n}, w>_{H^{-1}(\Omega), H_{0}^{1}(\Omega)}+\int_{\Omega} y^{n} \cdot \nabla y^{n} \cdot w, \quad \forall w \in \boldsymbol{V} .
\end{aligned}
$$

For $\delta t$ of the order $10^{-3}$, this first-order in time scheme displays, for $\nu=1 / 1000$ and the semi-disk geometry 4 , very similar results to the conditionally stable partially explicit scheme (we refer to 
[19, Section 5.1])

$$
\int_{\Omega} \alpha y^{n+1} \cdot w+\nu \nabla y^{n+1} \cdot \nabla w+y^{n} \cdot \nabla y^{n} \cdot w=\int_{\Omega} \alpha y^{n} \cdot w+<f^{n}, w>_{H^{-1}(\Omega), H_{0}^{1}(\Omega)}, \quad \forall w \in \boldsymbol{V}
$$

and to unconditionally stable scheme

$$
\int_{\Omega} \alpha y^{n+1} \cdot w+\nu \nabla y^{n+1} \cdot \nabla w+y^{n} \cdot \nabla y^{n+1} \cdot w=\int_{\Omega} \alpha y^{n} \cdot w+<f^{n}, w>_{H^{-1}(\Omega), H_{0}^{1}(\Omega)}, \quad \forall w \in \boldsymbol{V} .
$$

In term of computational cost, the scheme (4.4) is as expected faster than the scheme 4.3 : the ratio of the computational time to perform 10000 iterates (leading to $T=10$ ) between 4.3 and (4.4) is approximatively equal to 1.65. The regular triangulation used corresponds to a mesh size $h$ of the order of $6.23 \times 10^{-3}$ making 4.4 stable. On the other hand, the computational times of (4.3) and 4.5 are equivalent: we observe a ratio equal to 1.05. For $\delta t=10^{-2}$, scheme (4.4) is unstable. The convergence with respect to $k$ of 4.2 (no more equivalent to 4.3) is observed after two iterates. The ratio of computational time between 4.2) and 4.5 raises to 1.89. We observe that the approximation for 4.2 is much less sensitive to the variation of $\delta t$ : we observe the same result than with $\delta t=10^{-3}$ and than [5] where $\delta t=5 \times 10^{-4}$ is used.

\section{Conclusions And Perspectives}

We have rigorously analyzed a weak least-squares method introduced forty years ago in [2] allowing to solve a steady nonlinear Navier-Stokes equation, in the incompressible regime. This equation with a zero order term appears after any fully implicit time discretization of the unsteady Navier-Stokes equation. We have constructed a sequence converging strongly to the solution of the steady equation. Using a particular descent direction very appropriate for the analysis, this convergent sequence turns out to coincide with the sequence obtained using the damped Newton method to solve the underlying variational weak formulation. This globally convergent approach enjoys a quadratic rate a convergence after a finite number of iterates and is in particular much faster than the conjugate gradient method used in [2, 11]. Then, we have shown the convergence of the method, uniformly with respect to the time discretization, to solve the fully implicitly Euler scheme associated to the unsteady Navier-Stokes equation. When the time discretization is fine enough, each step of the damped Newton method simply reduced to the Newton one. In a such a case, we obtained a proof of convergence of the Newton scheme to solve the unsteady Navier-Stokes. As far as we know, this proof is original. Numerical experiments have highlighted the robustness of the method, even for small values of the viscosity coefficient. We also emphasize that the least-squares approaches, employed here to treat the Navier-Stokes nonlinearity, can be used to solve another nonlinear equations, as formally done in [14 for a sublinear heat equation. Eventually, we may solve the unsteady Navier-Stokes system by a fully $L^{2}\left(0, T ; H^{-1}(\Omega)\right)$ leastsquares approach. The underlying corrector solves an unsteady Stokes type equation; we refer to $[12]$.

\section{REFERENCES}

1. Pavel B. Bochev and Max D. Gunzburger, Least-squares finite element methods, Applied Mathematical Sciences, vol. 166, Springer, New York, 2009. MR 2490235

2. M. O. Bristeau, O. Pironneau, R. Glowinski, J. Periaux, and P. Perrier, On the numerical solution of nonlinear problems in fluid dynamics by least squares and finite element methods. I. Least square formulations and conjugate gradie, Comput. Methods Appl. Mech. Engrg. 17/18 (1979), no. part 3, 619-657. MR 529979

3. Peter Deuflhard, Newton methods for nonlinear problems, Springer Series in Computational Mathematics, vol. 35, Springer, Heidelberg, 2011, Affine invariance and adaptive algorithms, First softcover printing of the 2006 corrected printing.

4. Vivette Girault and Pierre-Arnaud Raviart, Finite element methods for Navier-Stokes equations, Springer Series in Computational Mathematics, vol. 5, Springer-Verlag, Berlin, 1986, Theory and algorithms. MR 851383 
5. R. Glowinski, G. Guidoboni, and T.-W. Pan, Wall-driven incompressible viscous flow in a two-dimensional semi-circular cavity, J. Comput. Phys. 216 (2006), no. 1, 76-91. MR 2223437

6. Roland Glowinski, Finite element methods for incompressible viscous flow, Numerical Methods for Fluids (Part 3), Handbook of Numerical Analysis, vol. 9, Elsevier, 2003, pp. 3 - 1176.

7. Roland Glowinski, Variational methods for the numerical solution of nonlinear elliptic problems, CBMS-NSF Regional Conference Series in Applied Mathematics, vol. 86, Society for Industrial and Applied Mathematics (SIAM), Philadelphia, PA, 2015. MR 3450066

8. F. Hecht, New development in freefem ++ , J. Numer. Math. 20 (2012), no. 3-4, 251-265. MR 3043640

9. Bo-Nan Jiang and Louis A. Povinelli, Least-squares finite element method for fluid dynamics, Comput. Methods Appl. Mech. Engrg. 81 (1990), no. 1, 13-37. MR 1071091

10. Sang Dong Kim, Yong Hun Lee, and Byeong Chun Shin, Newton's method for the Navier-Stokes equations with finite-element initial guess of Stokes equations, Comput. Math. Appl. 51 (2006), no. 5, 805-816. MR 2224683

11. J. Lemoine, A. Munch, and P. Pedregal, Analysis of continuous $H^{-1}$-least-squares approaches for the steady Navier-Stokes system, To appear in Applied Mathematics and Optimization (2020).

12. Jérome Lemoine and Arnaud Münch, A fully space-time least-squares method for the unsteady Navier-Stokes system, Preprint. arXiv:1909.05034.

13. J.-L. Lions, Quelques méthodes de résolution des problèmes aux limites non linéaires, Dunod; GauthierVillars, Paris, 1969. MR 0259693

14. Arnaud Münch and Pablo Pedregal, Numerical null controllability of the heat equation through a least squares and variational approach, European J. Appl. Math. 25 (2014), no. 3, 277-306. MR 3197994

15. Alfio Quarteroni and Alberto Valli, Numerical approximation of partial differential equations, Springer Series in Computational Mathematics, vol. 23, Springer-Verlag, Berlin, 1994. MR 1299729

16. Pierre Saramito, A damped Newton algorithm for computing viscoplastic fluid flows, J. Non-Newton. Fluid Mech. 238 (2016), 6-15. MR 3577347

17. Antony Smith and David Silvester, Implicit algorithms and their linearization for the transient incompressible Navier-Stokes equations, IMA J. Numer. Anal. 17 (1997), no. 4, 527-545. MR 1476337

18. Luc Tartar, An introduction to Navier-Stokes equation and oceanography, Lecture Notes of the Unione Matematica Italiana, vol. 1, Springer-Verlag, Berlin; UMI, Bologna, 2006. MR 2258988

19. Roger Temam, Navier-Stokes equations, AMS Chelsea Publishing, Providence, RI, 2001, Theory and numerical analysis, Reprint of the 1984 edition. MR 1846644

20. F. Tone and D. Wirosoetisno, On the long-time stability of the implicit Euler scheme for the two-dimensional Navier-Stokes equations, SIAM J. Numer. Anal. 44 (2006), no. 1, 29-40. MR 2217369 